\title{
Diffusion-weighted magnetic resonance spectroscopy enables cell-specific monitoring of astrocyte reactivity in vivo
}

\author{
Clémence Ligneul $^{\mathrm{a}, \mathrm{b}}$, Marco Palombo ${ }^{\mathrm{c}, 1}$, Edwin Hernández-Garzón ${ }^{\mathrm{a}, \mathrm{b}, 1}$, \\ María-Angeles Carrillo-de Sauvage ${ }^{\mathrm{a}, \mathrm{b}}$, Julien Flament ${ }^{\mathrm{a}, \mathrm{d}}$, Philippe Hantraye ${ }^{\mathrm{a}, \mathrm{b}, \mathrm{d}}$, \\ Emmanuel Brouillet $^{\mathrm{a}, \mathrm{b}}$, Gilles Bonvento ${ }^{\mathrm{a}, \mathrm{b}}$, Carole Escartin ${ }^{\mathrm{a}, \mathrm{b}}$, Julien Valette ${ }^{\mathrm{a}, \mathrm{b},{ }^{\prime \prime}}$ \\ ${ }^{a}$ Commissariat à l'Energie Atomique et aux Energies Alternatives (CEA), MIRCen, F-92260, Fontenay-aux-Roses, France \\ ${ }^{\mathrm{b}}$ Centre National de la Recherche Scientifique (CNRS), Université Paris-Sud, Université Paris-Saclay, UMR 9199, Neurodegenerative Diseases Laboratory, F-92260, \\ Fontenay-aux-Roses, France \\ ${ }^{c}$ Department of Computer Science and Centre for Medical Image Computing, University College of London, London, WC1E 6BT, United Kingdom \\ ${ }^{\mathrm{d}}$ Institut National de la Santé et de le Recherche Médicale (INSERM), Unité Mixte de Service UMS 27, F-92260, Fontenay-aux-Roses, France
}

\section{A B S T R A C T}

Reactive astrocytes exhibit hypertrophic morphology and altered metabolism. Deciphering astrocytic status would be of great importance to understand their role and dysregulation in pathologies, but most analytical methods remain highly invasive or destructive. The diffusion of brain metabolites, as non-invasively measured using diffusion-weighted magnetic resonance spectroscopy (DW-MRS) in vivo, depends on the structure of their micro-environment. Here we perform advanced DW-MRS in a mouse model of reactive astrocytes to determine how cellular compartments confining metabolite diffusion are changing. This reveals myo-inositol as a specific intraastrocytic marker whose diffusion closely reflects astrocytic morphology, enabling non-invasive detection of astrocyte hypertrophy (subsequently confirmed by confocal microscopy ex vivo). Furthermore, we measure massive variations of lactate diffusion properties, suggesting that intracellular lactate is predominantly astrocytic under control conditions, but predominantly neuronal in case of astrocyte reactivity. This indicates massive remodeling of lactate metabolism, as lactate compartmentation is tightly linked to the astrocyte-to-neuron lactate shuttle mechanism.

\section{Introduction}

The brain is an organ of immense complexity, whose function relies on the interactions of different cell types, each with their own morphological and metabolic specificities. Astrocytes are emerging as key players in the major brain function, i.e. transmission through neuronal networks, via fine synaptic modulation, synaptic connectivity establishment and spread of synaptic signalization (Araque et al., 2014; Navarrete and Araque, 2014). Moreover, given that almost all brain functions are essentially regulated by glial cells, there is an increasing awareness of their importance in the whole spectrum of neurological disorders (Zuchero and Barres, 2015). Reactive astrocytes are a hallmark of many brain pathologies (Dossi et al., 2018), including neurodegenerative diseases (Ben Haim et al., 2015), and have been proposed to play a major role in synaptic plasticity (Tyzack et al., 2014; Haydon et Nedergaard, 2015; Pittà et al., 2016; Liddelow and Barres, 2017). They notably display a hypertrophic morphology (usually visualized with GFAP staining), and increased ketone bodies utilization to fuel increased oxidative metabolism (Escartin et al., 2007).

The quest for non-invasive tools capable of probing cellular status to better understand the normal brain, but also to characterize and monitor its dysregulations under pathological conditions, has been driving considerable methodological research. In particular, over the last 30 years, nuclear magnetic resonance has delivered revolutionary tools to non-invasively probe brain cell structure and metabolism. Magnetic resonance imaging (MRI), which relies on the detection of water molecules, is now widely used to investigate brain characteristics (anatomy, function and microstructure) via several types of contrasts, including diffusion MRI to inform about cellular structure. Indeed, water diffusion is sensitive to restrictions imposed by cellular membranes, thus indirectly informing on the underlying cellular structure (Alexander et al., 2017). In parallel, magnetic resonance spectroscopy (MRS), where the signal originates from metabolites resonating at different frequencies than water, offers unparalleled access to cellular metabolism. Some

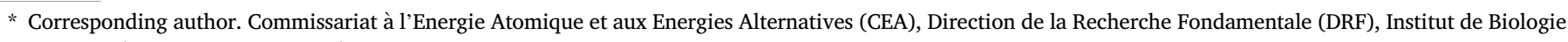
François Jacob, MIRCen, 18 Route du Panorama, 92260, Fontenay-aux-Roses, France.

E-mail address: julien.valette@cea.fr (J. Valette).

1 Authors contributed equally to this work.
} 
metabolites are confined in the intracellular space (in contrary to water), and some of them are preferentially found in particular cell-types. From works mostly based on cell cultures, N-acetylaspartate (NAA) and glutamate (Glu) have for example been reported with a higher concentration in neurons (Urenjak et al., 1993; Griffin et al., 2002), while choline compounds (tCho) and myo-inositol (Ins) are thought to be predominantly in glial cells (Gill et al., 1989). The diffusion of these metabolites, as measured using diffusion-weighted MRS (DW-MRS), mostly depends on the cellular structure constraining them (Ligneul et al., 2017; Palombo et al., 2017a).

Some recent works, including ours, report different diffusion behaviors among these metabolites in the healthy brain, which can be reasonably (and quantitatively) explained in terms of different cellular structure, yielding some additional evidence about their preferential cellular specificity (see Palombo et al., 2017c for review). Nevertheless, brain metabolite compartmentation is still an open topic of discussion, since its investigation is highly challenging. On one side, ex vivo immunochemistry approaches lack metabolite-specific markers and conventional euthanasia procedures alter the metabolism. On the other, metabolite quantification in specific cell culture or samples of sorted cells using MRS or other analytical techniques might not fully reflect the in vivo conditions. Independently of the specific compartmentation of these metabolites, it has never been established that variations of their diffusion, e.g. as observed in some diseases (Wood et al., 2012; Ercan et al., 2016), could indeed be linked to alterations of cellular morphology.

In this work, we aim at clarifying the ability of DW-MRS to distinguish metabolite diffusing in different cellular compartments, and thus to noninvasively assess metabolic compartmentation and cell structure. We investigate a mouse model of "pure" astrocyte reactivity induced by the cytokine ciliary neurotrophic factor (CNTF), with astrocytes becoming hypertrophic while neither neuronal death nor microglial activation are detected (Escartin et al., 2006, 2007). Our primary working hypothesis is that intracellular metabolites preferentially concentrated in astrocytes would exhibit diffusion alterations that are quantitatively consistent with astrocytic hypertrophy as measured by confocal microscopy, while other intracellular metabolites, in particular metabolites mostly found in neurons, would exhibit negligible or no diffusion alteration. It turns out that, among the intracellular metabolites, only myo-inositol exhibits significant diffusion alterations, which can indeed be explained by astrocytic hypertrophy. Beyond that, we also pay particular attention to lactate, which, unlike other metabolites, is found at significant levels in both intracellular and extracellular spaces. We observe massive variation of lactate diffusion, in a way that suggests a modification in its cellular compartmentation, with intracellular lactate being predominantly astrocytic in control mice, but becoming predominantly neuronal in CNTF mice.

\section{Methods}

\subsection{Mouse model of astrocyte reactivity}

All experimental protocols were reviewed and approved by the local ethics committee (CETEA $\mathrm{N}^{\circ} 44$ ) and submitted to the French Ministry of Education and Research (approval: APAFIS\#4554-2016031709173407). They were performed in a facility authorized by local authorities (authorization \#B92-032-02), in strict accordance with recommendations of the European Union (2010-63/EEC). All efforts were made to minimize animal suffering and animal care was supervised by veterinarians and animal technicians. Mice were housed under standard environmental conditions (12-h light-dark cycle, temperature: $22 \pm 1{ }^{\circ} \mathrm{C}$ and humidity: 50\%) with ad libitum access to food and water. Selfinactivated lentiviral vectors (LV) pseudotyped with the vesicular stomatitis virus envelope were produced and titrated as described in (Escartin et al., 2006). They encode the human ciliary neurotrophic factor (CNTF) cDNA with the immunoglobulin export sequence or $\beta$-galactosidase (LacZ) cDNA under the phosphoglycerate kinase I promoter, and specifically target neurons. To visualize astrocyte morphology with confocal laser microscopy, we used LV encoding green fluorescence protein (GFP) and pseudotyped with the Mokola envelope to target astrocytes. These LV also bear the Mir124 target sequence to repress GFP expression in neurons (Colin et al., 2009). Two-month-old male C57bl6 mice (Charles River, France) were anesthetized with an i.p. injection of ketamine $(100 \mathrm{mg} / \mathrm{kg})$ and xylazine $(10 \mathrm{mg} / \mathrm{kg})$. Lidocaine $(7 \mathrm{mg} / \mathrm{kg})$ was injected subcutaneously at the incision site, $10 \mathrm{~min}$ before injection. LV were diluted in PBS with $1 \%$ bovine serum albumin. Three types of striatal injections were performed: 1) bilateral $1 \mu$ l-injections of LV-CNTF ( $83 \mathrm{ng}$ of p24 per site), performed on ten mice forming the "CNTF group" for MRS experiments; 2) bilateral $1 \mu$ l-injections of LV-LacZ (83 ng of p24 per site), performed on ten mice forming the "control group" for MRS experiments; and 3) $1.4 \mu$ l-injections of LV-LacZ + LV-GFP in the left striatum and LV-CNTF + LV-GFP in the right striatum (83 ng of p24 of LV-CNTF or LV-LacZ + 17 ng p24 LV-GFP), performed on five mice for confocal microscopy. For MRS experiments, we decided to inject bilaterally to maximize voxel size (and signal-to-noise ratio). For confocal microscopy experiments, since one hemisphere by animal could bring the necessary information, we decided to inject unilaterally to minimize the amount of animals to sacrifice. Anesthetized mice were placed on the stereotactic frame and were injected with $\mathrm{LV}$ dilutions at a rate of $0.2 \mu \mathrm{l} / \mathrm{min}$ with the following coordinates: anteroposterior $+1 \mathrm{~mm}$; lateral $\pm 2 \mathrm{~mm}$ from bregma and ventral $-2.5 \mathrm{~mm}$ from the dura, tooth bar set at $0 \mathrm{~mm}$. At the end of injection, the needle was left in place for $5 \mathrm{~min}$ before being slowly removed and the skin was sutured. We showed previously that CNTF effects are stable over time (Escartin et al., 2006; Carrillo-de Sauvage et al., 2015).

\subsection{MRI and MRS experiments}

Mice were scanned 6 weeks after injection on an 11.7 T Bruker horizontal scanner running with Paravision 6.0.1 (Bruker, Ettlingen, Germany), with maximal gradient strength $G_{\max }=752 \mathrm{mT} / \mathrm{m}$ on each axis and $100-\mu$ s rise time. A quadrature surface cryoprobe (Bruker, Ettlingen, Germany) was used for radiofrequency transmission and reception. Animals were maintained on a stereotaxic bed with a bite and two ear bars. They were anesthetized with $1-1.3 \%$ isoflurane in a 1:1 mixture of air and oxygen $(1 \mathrm{~L} / \mathrm{min}$ total flow rate). Mouse body temperature was monitored with an endorectal probe and maintained at $37{ }^{\circ} \mathrm{C}$ with regulated water flow, and respiratory rate was continuously monitored using PC SAM software (Small Animal Instruments, Inc., Stony Brook, NY) during scanning.

A spectroscopic volume of interest $\left(6.5 \times 3 \times 2.8=56 \mathrm{~mm}^{3}\right)$ was placed around the injection site in the striatum (Fig. 1A), and signal was acquired with our recent STE-LASER sequence (Ligneul et al., 2017), designed to provide excellent localization while avoiding cross-terms between diffusion gradients and selection gradients. The echo time TE was $33.4 \mathrm{~ms}$, and diffusion gradient duration $\delta$ was set at $3 \mathrm{~ms}$. Water suppression was achieved with a VAPOR module and an additional water suppression pulse at the end of the mixing time. The full set of DW-MRS measurements was performed in each mouse. First, spectra at different diffusion-weightings ( $b=0.02,3.02,6,10,20,30$ and $50 \mathrm{~ms} / \mu \mathrm{m}^{2}, \mathrm{TR}=2000 \mathrm{~ms}, 128$ repeats) were acquired at $t_{d}=53.2 \mathrm{~ms}$. Then, spectra were acquired at longer $t_{d}$ for two different $b$-values: $b_{0}$ corresponding to the minimal diffusion-weighting achievable while keeping sufficient diffusion gradient strength for efficient spoiling, and $b=b_{0}+3 \mathrm{~ms} / \mu \mathrm{m}^{2}$ to extract the apparent diffusion coefficient of metabolites according to ADC $=-\frac{1}{b-b 0} \ln \left(\frac{s}{s_{0}}\right)$. TR was different for the different $t_{d}$ to allow for similar recovery delay after the LASER localization block. The number of repetitions was increased at the two longest diffusion times to get sufficient SNR. Acquisition parameters were $t_{d}$ $(\mathrm{ms}) / \mathrm{TR}(\mathrm{ms}) / b_{0}\left(\mathrm{~ms} / \mu \mathrm{m}^{2}\right) /$ number of repetitions $=253.2 / 2200 / 0.1 / 128$, $503.2 / 2500 / 0.2 / 128,1003.2 / 3000 / 0.3 / 128,1503.2 / 35000 / 0.45 / 512$, 2003.2/4000/0.6/512. A macromolecule spectrum was acquired for each 


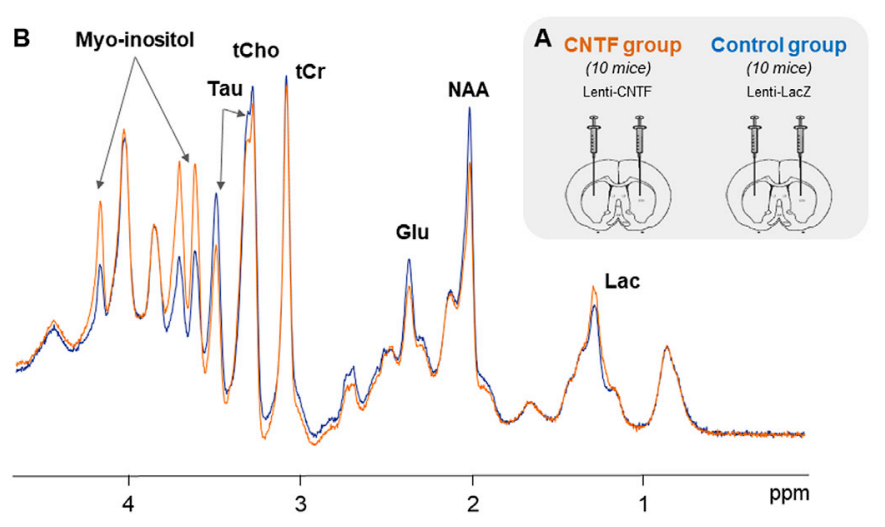

C

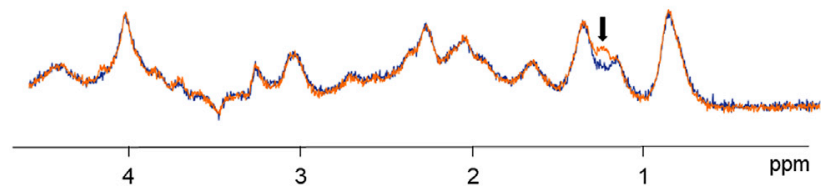

Fig. 1. Alterations detected in the CNTF mouse model of reactive astrocytes using in vivo MRS. (A) Astrocyte reactivity was induced in the striatum by bilateral injection of lentiviral vectors specifically targeting neurons and encoding for CNTF, or $\beta$-galactosidase (LacZ) for control. (B) Average of all spectra acquired in each group (10 mice per group) to illustrate variations of metabolite levels $(\mathrm{Glu}=$ glutamate, Lac $=$ lactate, $\mathrm{Tau}=$ taurine, $\mathrm{tCho}=$ choline compounds, $\mathrm{tCr}=$ total creatine). (C) Average of macromolecule spectra acquired in each groups. These spectra are very similar except for the small peak appearing at $\sim 1.2 \mathrm{ppm}$, likely corresponding to $\beta$-hydroxybutyrate. Neither scaling nor filtering was applied to spectra.

mouse ( $\mathrm{TR}=4000 \mathrm{~ms}, 256$ averages). In addition to a double inversion recovery module (TI1 $=2200 \mathrm{ms,} \mathrm{TI2}=730 \mathrm{~ms}$ ) placed prior to the STE-LASER sequence, $b=10 \mathrm{~ms} / \mu \mathrm{m}^{2}$ was applied to reduce residual metabolite peaks. Diffusion MRI was also performed to evaluate its sensitivity to astrocyte reactivity. Images were acquired using 2D spin-echo EPI (TE/TR $=30 / 3200 \mathrm{~ms}$, six segments, 400-kHz acquisition bandwidth), with an $18 \times 18 \mathrm{~mm}^{2}$ field-of-view spanned by $160 \times 160$ pixels and ten 0.5 -mm slices. Diffusion gradient duration $\delta$ was $2 \mathrm{~ms}$ and gradient separation $\Delta$ was $20 \mathrm{~ms}$. In addition to the five $\mathrm{b}=0$ images, thirty gradient directions were acquired at two $b$-values ( $1 \mathrm{~ms} / \mu \mathrm{m}^{2}$ and $2 \mathrm{~ms} / \mu \mathrm{m}^{2}$ ) to allow for tensor (DTI) and Kurtosis (DKI) analysis. Fractional anisotropy (FA) and mean diffusivity (MD) maps could be reconstructed from DTI and DKI analysis (Fig. S1). Kurtosis map was also reconstructed from DKI. A region of interest was drawn in the striatum to compute the average value for each of these parameters.

\subsection{MRS processing and quantification}

Scan-to-scan phase correction was performed on metabolite signal before summing individual scans on Matlab, to correct for incoherent averaging leading to artefactual signal loss. Eddy current correction was achieved using water reference. Spectra were analyzed with LCModel (Provencher, 1993), using a basis set generated for each TM with home-made routines based on the density matrix formalism. Signal could be reliably quantified according to our quality standards (Cramér-Rao lower bounds $\mathrm{CRLB}<5 \%$ ) for NAA, tCr, tCho, Glu, Ins, and Tau at all $b$-values and $\mathrm{t}_{\mathrm{d}}$. Individual macromolecule spectra acquired in mice were summed with respect to their group and then included in LCModel basis sets associated to the CNTF and control groups. Metabolite concentrations were evaluated from spectra acquired at $\mathrm{t}_{\mathrm{d}}=53.2 \mathrm{~ms} / b=0.020 \mathrm{~ms} / \mu \mathrm{m}^{2}$. For this parameter set NAA, tCr, tCho, Glu, Ins, Tau, Gln, GABA and Lac were reliably quantified (CRLB $<5 \%$ ). As a preliminary check, we summed all spectra of the control group and all spectra of the CNTF group to search for a stable metabolite in both groups to serve as internal reference. Absolute $\mathrm{tCr}$ signal was found to be very stable between both groups (Fig. 1B), therefore we used it as an internal reference standard (fixed at $8 \mathrm{mM}$ in both groups) for quantification of metabolite concentration in all individual spectra. Absolute macromolecule signal (peak at $0.9 \mathrm{ppm}$ ) also appeared to be very stable (see Fig. 1C), confirming the stability of the amount of tissue in the volume of interest.

\subsection{Statistical analysis of MRS data}

For metabolite levels measured in MRS experiments, statistical significance of the effect of the group (CNTF or control) on metabolites concentrations (only those quantified with Cramér-Rao lower bounds $<5 \%$ ) was assessed by an unpaired Student's t-test. To compensate for the type I error due to multiple comparisons, p-values were adjusted by a Bonferroni correction over the eight metabolites considered. In DW-MRS experiments, we wanted to evaluate the statistical significance of the effect of the group (CNTF or control) on the functional form of the diffusion, i.e. signal attenuation over $b$ or on $\mathrm{ADC}$ over $\mathrm{t}_{\mathrm{d}}$, as this functional form carries the information about the underlying microstructure. This was assessed by an analysis of variance (ANOVA) with one betweensubject factor (group CNTF or control) and one within-subject factor ( $b$ value or $t_{d}$ ). Data homoscedasticity was confirmed by a Barlett test. After identifying intracellular metabolites exhibiting a significant difference in diffusion properties between groups, i.e. a group* $b$ or group* $t_{d}$ interaction (which was the case only for myo-inositol), we ran unpaired Student's t-test as post hoc tests on each value of the within-subject factor, i.e. on each point of myo-inositol diffusion curves (signal attenuation as a function of $b$ or ADC as a function of $t_{d}$ ). To compensate for the type I error due to multiple comparisons over the six levels of each factor (six non-zero $b$-values, and six $\mathrm{t}_{\mathrm{d}}$ ), $\mathrm{p}$-values were adjusted by a Bonferroni correction with $n=6$.

\subsection{DTI and DKI analysis}

Diffusion-weighted images were denoised using MP-PCA as described in (Veraart et al., 2016), corrected for Gibbs ringing artifacts following (Kellner et al., 2016) and for eddy-currents artifacts using FSL eddy (Andersson and Sotiropoulos, 2016). No artifacts due to brain motion were detected. DTI (Basser et al., 1994; Basser and Pierpaoli, 1996) and DKI (Jensen et al., 2005; Tabesh et al., 2011) analyses were performed using home-made script in python, harnessing the DIPY's (Garyfallidis et al., 2014) DTI and DKI modules employing weighted least square minimization to voxel-wise estimate the 6 and 15 independent components of the diffusion (DT) and kurtosis (KT) tensors, respectively. From the DT, standard diffusion statistics such as mean diffusivity (MD) and fractional anisotropy (FA) were computed, as described in Basser and Pierpaoli (1996). Since the diffusion statistics from the kurtosis model are expected to have better accuracy (Veraart et al., 2011), MD and FA were also computed from the KT, together with non-Gaussian statistics such as mean kurtosis (MK), as described in the study of (Tabesh et al., 2011).

\subsection{Diffusion modeling for analysis of high-b data to evaluate the radius of fibers and soma}

To analyze the DW-MRS data and estimate the radius of cellular soma and projections, we used our recently introduced cylinder model (Palombo et al., 2017a), with a small correction to account for soma contribution, as recently proposed in (Palombo et al., 2018a, 2018b). Specifically, cellular projections can be described in first approximation as a collection of long cylinders (the "fibers"), with radius $\mathrm{R}_{\mathrm{fiber}}$, and intracellular diffusivity $\mathrm{D}_{\text {intra. }}$. We assume that cylinders are randomly oriented to calculate the signal attenuation $S_{\text {fiber }}$ in the narrow pulse approximation, as described in (Palombo et al., 2017a). The signal represents the sum of signals from a large number of differently oriented 
fibers. For any given fiber, the axis makes a variable angle $\theta$ with the diffusion gradient, leading to two diffusion regimes: i) restricted diffusion in the plane perpendicular to their axis, with an effective gradient strength $g_{\perp}=g \sin (\theta)$ and ii) free diffusion in the plane parallel to their axis, with an effective gradient strength $g_{\|}=g \cos (\theta)$.

When gradients are in the direction $\theta$ to the axis of the cylinder of radius $R_{\text {fiber }}$, the echo attenuation is given by the following expression (Linse and Soderman, 1995):

$$
\begin{aligned}
E_{\text {fiber }}\left(\theta, R_{\text {fiber }}, D_{\text {intra }}, g, \delta, t_{d}\right)= & \left\{\frac{\left[2 J_{1} \gamma g_{\perp} \delta R_{\text {fiber }}\right]^{2}}{\left(\gamma g_{\perp} \delta R_{\text {fiber }}\right)^{2}}\right. \\
& \left.+8\left(\gamma g_{\perp} \delta R_{\text {fiber }}\right)^{2} A_{n m}\right\} e^{-D_{\text {intra }}\left(\gamma g_{\|} \delta\right)^{2} t_{d}}
\end{aligned}
$$

with with $\mathrm{S}_{\text {fiber }}$ and $\mathrm{S}_{\text {soma }}$ given by equations [1] and [2], respectively.

Equation (3) was fitted to myo-inositol signal attenuation as a function of $b$, as measured in vivo and averaged over all animals from each group, to estimate three free parameters: $R_{\text {soma }}, R_{\text {fiber }}$ and $D_{\text {intra. }}$.

Fitting was performed by using a nonlinear least-square regression, based on the trust-region-reflective algorithm implemented in MATLAB (The MathWorks, Natick, Massachusetts, USA). The error on estimated parameters was evaluated using a Monte Carlo (MC) approach ( $\mathrm{n}=2500$ draws). For each draw, random noise (whose standard deviation s.d. was estimated from the difference between the best fit and the experimental data) was generated and added to the best fit to generate a new data set, which could be analyzed using the model. Statistical difference between control and CNTF values was assessed by permutation analysis of the parameter distribution estimated from the $2500 \mathrm{MC}$ draws using unpaired two-tailed $t$-test (with Bonferroni correction for the two structural parameters of interest $\mathrm{R}_{\text {fiber }}$ and $\mathrm{R}_{\text {soma }}$ ).

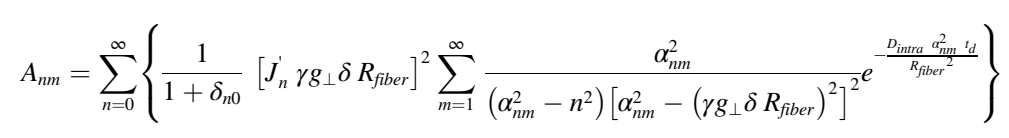

where the dependence on $\theta$ is implicitly expressed by $g_{\perp}=g \sin (\theta)$ and $g_{\|}=g \cos (\theta), J_{n}$ is the Bessel function of integer order $\mathrm{n}$ and $\alpha_{n m}$ is the mth positive root of the Bessel equation $J_{n}^{\prime}=0 . \delta_{n 0}$ is the Kronecker delta symbol. The total signal $\mathrm{S}_{\text {fiber }}$ is then computed as discretized approximation of the integral over all directions:

$S_{\text {fiber }}\left(R_{\text {fiber }}, D_{\text {intra }}, g, \delta, t_{d}\right)=\frac{\int_{0}^{\pi / 2} d \theta \sin (\theta) E_{\text {fiber }}\left(\theta, R_{\text {fiber }}, D_{\text {intra }}, g, \delta, t_{d}\right)}{\int_{0}^{\pi / 2} d \theta \sin (\theta)}$

Furthermore, we consider the cellular volume fraction occupied by astrocytic processes to be $f$, the remaining intracellular fraction $1-f$ being occupied by the soma. Studies carried on different species and with different methods (Chvatal et al., 2007) report fibers representing $80-85 \%$ of the total astrocytic volume. Our own confocal measurements (see details below) yield $f \sim 77 \%$ in control and $\sim 71 \%$ in CNTF. In line with these estimates, in our model we set $f=80 \%$. The DW-MRS signal originating from cellular soma, $\mathrm{S}_{\text {soma }}$, is modeled as the signal arising from molecular diffusion restricted within a sphere (Linse and Soderman, 1995 ) of radius $R_{\text {soma }}$ and intracellular diffusivity $D_{\text {intra, }}$, and it contributes to the total DW-MRS signal S with a weight $1-f=20 \%$, in our model. Specifically, the signal for diffusion within a sphere of radius $R_{\text {soma }}$ in the narrow pulse approximation is (Linse and Soderman, 1995):

$S_{\text {soma }}\left(R_{\text {soma }}, D_{\text {intra }}, g, \delta, t_{d}\right)=\frac{\left[3 j_{1}\left(\gamma g \delta R_{\text {soma }}\right)\right]^{2}}{\left(\gamma g \delta R_{\text {soma }}\right)^{2}}+6\left(\gamma g \delta R_{\text {soma }}\right)^{2} \mathrm{~B}_{n m}$

with
2.7. Diffusion modeling and machine learning for analysis of long-td data and evaluation of long-range cellular morphology

The ADC time-dependency of brain metabolites was analyzed using the computational framework introduced in (Palombo et al., 2016). Briefly, cellular fibers are modeled as mono-dimensional branched objects embedded in a three-dimensional space, dubbed "cell-graphs". Here only two morphometric parameters were let as free parameters: the average number of successive embranchments (bifurcations) $\mathrm{N}_{\text {branch }}$ along each process, and the average segment length $\mathrm{L}_{\text {segment }}$ for a given segment of process comprised between two successive branching points. For each of these statistics, a Gaussian distribution was assumed. To limit the degrees of freedom, we fixed the standard deviations on $\mathrm{N}_{\text {branch }}$ and $\mathrm{L}_{\text {segment }}\left(\mathrm{SD}_{\mathrm{Nbranch}}=2\right.$ and $\left.\mathrm{SD}_{\mathrm{Lsegment}}=10 \mu \mathrm{m}\right)$, since the effect on $\operatorname{ADC}\left(t_{d}\right)$ is much smaller than the effect of mean $\mathrm{N}_{\text {branch }}$ and $\mathrm{L}_{\text {segment }}$. The number of processes radiating from the soma has no effect on ADC time-dependency (Palombo et al., 2016), and was therefore not varied (fixed to $5 \pm 2$ ). Note that, when generating the synthetic cells to build the dictionary, the angles between successive segments were randomly drawn between $-\pi / 2$ and $\pi / 2$.

Unlike in (Palombo et al., 2016), a dictionary-based approach was used here for better computational efficiency. To generate the dictionary, comprised of 8000 simulated $\mathrm{ADC}\left(\mathrm{t}_{\mathrm{d}}\right)$ curves, a large set of different synthetic cells (2048 synthetic cells) was generated for any given set of morphometric statistics $\left(\mathrm{N}_{\text {branch }}\right.$ and $\left.\mathrm{L}_{\text {segment }}\right)$, and diffusion of 2000 particles was then simulated in each cell according to a Monte Carlo algorithm, for different $D_{\text {intra }}$ values. The corresponding

$B_{n m}=\sum_{n=0}^{\infty}\left\{\left[j_{n}^{\prime} \gamma g \delta R_{\text {soma }}\right]^{2} \sum_{m=1}^{\infty} \frac{(2 n+1) \alpha_{n m}^{2}}{\left(\alpha_{n m}^{2}-n^{2}-n\right)\left[\alpha_{n m}^{2}-\left(\gamma g \delta R_{\text {soma }}\right)^{2}\right]^{2}} e^{-\frac{D_{\text {intra }} a_{n m}^{2} t_{d}}{R_{\text {soma }}^{2}}}\right\}$

where $\mathrm{j}_{\mathrm{n}}(\mathrm{x})$ is the spherical Bessel function of the first kind and $\alpha_{\mathrm{nm}}$ is the $m$ th positive root of the Bessel equation $\mathrm{j}_{\mathrm{n}}=0$.

In the end, the total signal as a function of $b=(\gamma g \delta)^{2} t_{d}$, where $\gamma$ is the proton gyromagnetic ratio, is:

$\mathrm{S}(b)=0.8 \times \mathrm{S}_{\text {fiber }}\left(R_{\text {fiber }}, D_{\text {intra }}, b\right)+0.2 \times \mathrm{S}_{\text {soma }}\left(R_{\text {soma }}, D_{\text {intra }}, b\right)$ diffusion-weighted signal was computed using the phase accumulation approach and summed over the whole set of cell-graphs to obtain the coarse-grained $\operatorname{ADC}\left(t_{d}\right)$. An efficient code was implemented in Matlab to parallelize this huge amount of computation on a GPU device (NVIDIA Tesla K20c). The whole procedure required about $3 \mathrm{~min}$ for each set of morphometric parameters, and about $400 \mathrm{~h}$ to build the full database by 
varying $\mathrm{D}_{\text {intra, }}$ mean $\mathrm{N}_{\text {branch }}$ and mean $\mathrm{L}_{\text {segment }}$.

To learn the mapping between the substrate parameters and ADC time-dependency, a random forest regression was used (Nedjati-Gilani et al., 2017). We used a widely employed and freely available random forest regressor in the sci-kit learn python toolkit (Pedregosa et al., 2011) with 100 trees and a maximum depth of 20 (preliminary experiments found that we obtain diminishing returns in accuracy above these values). More in-depth details of the implementation are available on the scikit-learn website (http://scikit-learn.org/). The forest was then trained on 6400 simulated datasets, with the remaining 1600 previously unseen feature vectors used for testing. Following the training and testing stages, we used the forest regressor on the ADC time-dependency as measured in vivo (averaged over all animals of each group) to estimate $\mathrm{D}_{\text {intra }}, \mathrm{N}_{\text {branch }}$ and $\mathrm{L}_{\text {segment }}$ for myo-inositol compartment. At this point we should mention that conventional cell analysis tools do not provide a direct estimation of $\mathrm{N}_{\text {branch }}$, which is an ad hoc parameter used to control the branching complexity of our cell-graphs. To facilitate further comparison with real astrocytes as measured by microscopy (see next section), we found it more convenient to generate many cell-graphs corresponding to the set of $\left(\mathrm{N}_{\text {branch }}\right.$, $\left.\mathrm{L}_{\text {segment }}\right)$ values best explaining the data, and then, as for real cells, analyze these cell-graphs using the 3D Sholl analysis provided by the TREES Matlab toolbox to extract the "Branch Order" parameter characterizing the branching complexity (Cuntz et al., 2010). Stability of the estimated parameters with respect to experimental noise was evaluated using a Monte Carlo approach (2500 draws of noised datasets). At each Monte Carlo iteration, random Gaussian noise was generated with s.d. comparable to experimental s.d. on ADC $(\sim 15 \%)$ and added to the reference dataset to generate a new dataset, which was analyzed using the same pipeline. For each of the three parameters, a distribution of 2500 estimated values was obtained, and the best estimate and its uncertainty were then computed as the mean and s.d. of this distribution. Statistical difference between control and CNTF values was assessed by permutation analysis of the parameter distribution estimated from the $2500 \mathrm{MC}$ draws using unpaired two-tailed $t$-test (with Bonferroni correction for the two structural parameters of interest Branch Order and $\mathrm{L}_{\text {segment }}$ ).

\subsection{Confocal microscopy acquisitions, image analysis and astrocytes reconstruction}

Transcardial perfusion fixation of anesthetized mice, using cold $4 \%$ paraformaldehyde, was performed to get brain samples. Collected brains were kept at $4{ }^{\circ} \mathrm{C}$ in paraformaldehyde solution at least one night and then transferred to PBS. $100-120 \mu \mathrm{m}$ coronal sections of mouse brains were obtained with a vibrating-blade microtome (Leica) and then mounted in Vectashield mounting medium (Vector Labs). Isolated or small groups of astrocytes were imaged using a Leica SP8 confocal system equipped with a broadband white-light laser, with an oil immersion objective 63X, 1.4 NA. The excitation laser line was setup to $489 \mathrm{~nm}$ for eGFP excitation at $3 \%$ of intensity. Sequential scan mode imaging was used setting up the HyD photodetector to $498-570 \mathrm{~nm}$ for eGFP labeling. For all images a 1 airy unit pinhole was setup based on a 510 wavelength emission and the XY resolution and Z-step size was automatically optimized based on the Nyquist theorem. In all cases the white laser power was set to $70 \%$, and the acquisition speed of the laser scan was $600 \mathrm{~Hz}$.

Image analysis was performed using Fiji (Schindelin et al., 2012) Vaa3D (Peng et al., 2014), Matlab (MathWorks, USA), and Autoquant X3.1 (Media Cybernetics, USA). To reduce the workload due to the large size of original images $(\approx 4 \mathrm{~GB})$, regions of interest were cropped and each ROI was slice-aligned in the $\mathrm{X}$ and $\mathrm{Y}$ axis and deconvoluted using Autoquant X3.1, through the blind adaptive PSF method with ten iterations and low noise level. Then, images were adjusted in contrast using Fiji. Once in Vaa3D, the reconstruction of the astrocytes was made using the Vaa3D-Neuron2 Auto Tracing Based on APP2 plugin; basically, one marker was placed on the soma center of the single astrocyte and the plugin was run setting an auto-thresholding for the background, activating the option to get the radius from $2 \mathrm{D}$ and adjusting the length threshold until getting a proper reconstruction, all the other options were settled as default. Then, the structure was sorted using the "sort neuron" SWC plugin. To label the soma section, a six-face ROI was created around the user-defined volume and the segments inside that volume were labeled as soma, then each file was reviewed to correct wrong and missed segments. As Vaa3D only analyzes the SWC files in voxel units, the SWC were exported to Matlab using the TREES toolbox (Cuntz et al., 2010) and then the model was resized to the real dimensions from the initial acquisition. Finally, information from the model was extracted using L-measure (Scorcioni et al., 2008). The approach used to estimate the radius of the confocal reconstructions was to obtain the approximate circle of cross section area labeled in the XY plane based on the image for each marker of the reconstruction.

To analyze the long-range morphology of GFP-expressing astrocytes reconstructed by confocal microscopy, one should keep in mind that we are seeking a coherent comparison with synthetic cell-graphs estimated from long- $t_{d}$ data (the "synthetic astrocytes"). Hence it is necessary to work with objects exhibiting commensurate levels of details. To do so, prior to the morphology analysis with the TREES toolbox, cells reconstructed from confocal microscopy images were simplified, essentially by removing small secondary structures that are negligible in long- $t_{d}$ DWMRS experiments (Palombo et al., 2017b), and by "straightening" undulating segments (SI Appendix).

\section{Results}

\subsection{MRS identifies metabolic modifications associated with reactive astrocytes}

Astrocyte reactivity was induced in mice by intra-striatal injection of lentiviral vectors specifically targeting neurons and encoding for the human ciliary neurotrophic factor (CNTF), or $\beta$-galactosidase (LacZ) for control (Fig. 1A). We measured the metabolic profile of both phenotypes from spectra acquired at the lowest diffusion-weighting and shortest diffusion time. For visual inspection, the sum of spectra acquired in each individual mouse for both groups is shown in Fig. 1B, showing stable absolute levels of total creatine, but variations of concentration for many other metabolites. In the CNTF group, we report a lower concentration of NAA $(-20 \%)$, glutamate $(-17 \%)$ and taurine $(-18 \%)$, and a strong increase in myo-inositol concentration $(+92 \%)$ (Table 1$)$.

Lactate concentration may seem to slightly increase, but without significance. This metabolic remodeling is consistent with a previous MRS study conducted on rats injected with lenti-CNTF (Carrillo-de Sauvage et al., 2015), except for choline compounds that were increased in rats while they remain stable here. The macromolecule baseline is very similar between CNTF and control mice (Fig. 1C), except for an additional resonance around $1.2 \mathrm{ppm}$, likely corresponding to partly bound $\beta$-hydroxybutyrate. It is worth noting that the amplitude of macromolecule signal perfectly matches between both groups (except for this additional resonance at $1.2 \mathrm{ppm}$ ), underlining the absence of massive cellular loss or genesis in the MRS volume of interest, in line with the

Table 1

Metabolite concentrations in control and CNTF groups (mean \pm s.d, in mM), using $\mathrm{tCr}$ as an internal reference at $8 \mathrm{mM}$.

\begin{tabular}{llll}
\hline & Control & CNTF & $\begin{array}{l}\text { p-value (with Bonferroni } \\
\text { correction, } n=8 \text { ) }\end{array}$ \\
\hline Lac & $4.31 \pm 0.49$ & $4.78 \pm 0.70$ & 0.8 \\
Gaba & $2.64 \pm 0.42$ & $3.04 \pm 0.37$ & 0.3 \\
Gln & $3.20 \pm 0.18$ & $4.05 \pm 2.25$ & $>1$ \\
Glu & $\mathbf{7 . 4 9} \pm \mathbf{0 . 2 8}$ & $\mathbf{6 . 2 3} \pm \mathbf{0 . 4 0}$ & $\mathbf{2 e - 0 6}$ \\
Ins & $\mathbf{6 . 0 3} \pm \mathbf{0 . 2 6}$ & $\mathbf{1 1 . 6 2} \pm \mathbf{1 . 7 3}$ & $\mathbf{6 e - 0 8}$ \\
NAA & $\mathbf{7 . 3 4} \pm \mathbf{0 . 3 1}$ & $\mathbf{5 . 8 3} \pm \mathbf{0 . 4 6}$ & $\mathbf{7 e - 0 7}$ \\
Tau & $\mathbf{1 0 . 0 7} \pm \mathbf{0 . 3 1}$ & $\mathbf{8 . 2 9} \pm \mathbf{0 . 2 6}$ & $\mathbf{3 e - 1 0}$ \\
tCho & $2.52 \pm 0.19$ & $2.57 \pm 0.11$ & $>1$ \\
\hline
\end{tabular}


stability of striatal volume as measured from T2-weighed MRI images (31.6 $\pm 2.0 \mathrm{~mm}^{3}$ in CNTF group versus $31.4 \pm 1.6 \mathrm{~mm}^{3}$ in control group). Although we did not aim at quantifying absolute metabolites concentrations in this study, note that the relatively short TR used here leads to an underestimation of the taurine concentration $(\sim 20 \%)$ due to the longer T1 of this compound as compared to other metabolites, and in particular as compared to $\mathrm{tCr}$ used as reference.

\subsection{DW-MRS reveals myo-inositol as a specific intracellular diffusion marker of astrocyte reactivity}

In recent works performed in healthy animals, we have proposed that high diffusion-weighting DW-MRS data primarily reflect the radius of brain cell fibers (dendrites, axons, astrocytic processes ...) (Palombo et al., 2017b), while long diffusion times data primarily reflect fiber long-range structure, in particular complexity and length (Palombo et al., 2016). As discussed in (Palombo et al., 2017c), the diffusion of metabolites in brain cells can be modeled as long hollow cylinders when $t_{d}<100 \mathrm{~ms}$. Here we measured signal attenuation of reliable metabolites (CRLB $<5 \%$ ) up to a diffusion-weighting $b=50 \mathrm{~ms} / \mu \mathrm{m}^{2}$, at fixed diffusion time $t_{d}=53.2 \mathrm{~ms}$, which is therefore well suited to probe restriction in fibers' transverse plane. Among the six intracellular metabolites measured reliably, only myo-inositol exhibited significantly different signal attenuation curves from control (Fig. 2A and Table S1), with stronger attenuation in the CNTF group, suggesting that myo-inositol is diffusing inside fibers of larger radius. One can notice slightly stronger signal attenuation for tCho and slightly weaker NAA signal attenuation in the CNTF group, but these trends are not significant. As can be seen on Fig. 2A, macromolecules contribute a lot to high- $b$ spectra: the intensity of the broad macromolecules peak at $0.9 \mathrm{ppm}$ is comparable to NAA intensity. Therefore, it is crucial to properly quantify their signal for such experiments (Palombo et al., 2017c). Here the excellent spectral quality and signal-to-noise ratio SNR at all diffusion-weightings (at $b=50 \mathrm{~ms} / \mu \mathrm{m}^{2}$ the SNR, calculated relative to NAA, is $26 \pm 2$ in the CNTF group, and $30 \pm 2$ in the control group), as well as for macromolecules (Fig. 1C), allow reliable evaluation of the signal attenuation.

We then measured metabolite apparent diffusion coefficient (ADC) at long $t_{d}$ (up to $t_{d}=2003.2 \mathrm{~ms}$ ), leaving time for metabolites to explore the long-range fiber structure (Fig. 3). Again, only myo-inositol exhibited significant variation, with ADC decreasing less in the CNTF group as $t_{d}$ is increased (Fig. 3B and Table S1), indicating less long-range obstacles (which at first glance can be qualitatively interpreted as "longer fibers"). Spectral quality and SNR remain acceptable even in the least favorable situation (SNR $=28 \pm 4$ in CNTF group and $30 \pm 4$ in control group at $\mathrm{t}_{\mathrm{d}}=2003.2 \mathrm{~ms}$ and $b=3.6 \mathrm{~ms} / \mu \mathrm{m}^{2}$, calculated relative to NAA).

In the end, both high- $b$ and long- $t_{d}$ experiments reveal specific variations of myo-inositol diffusion in the CNTF group, which are qualitatively consistent with diffusion inside thicker and longer fibers, as a priori expected for hypertrophic reactive astrocytes. This strongly supports the idea that myo-inositol is compartmentalized in astrocytes, so that its diffusion reflects astrocytic morphology. Note that we also performed DW-MRI measurements, but no significant variation of related metrics (mean diffusivity, fractional anisotropy and mean kurtosis) were detected in the striatum (Fig. S1).
A

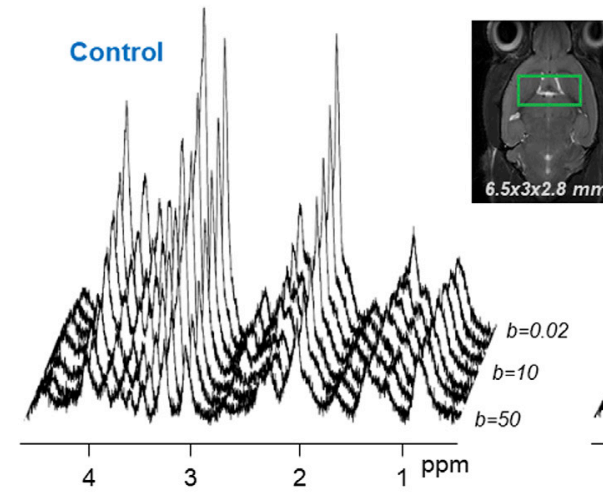

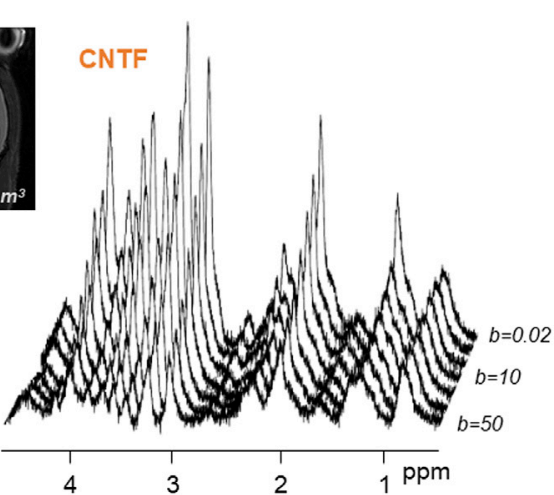

B

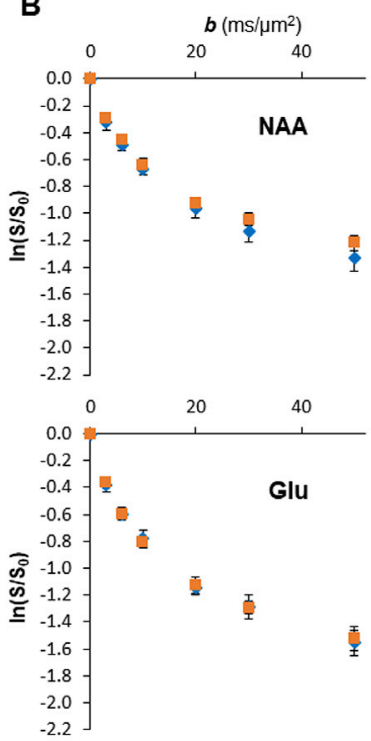

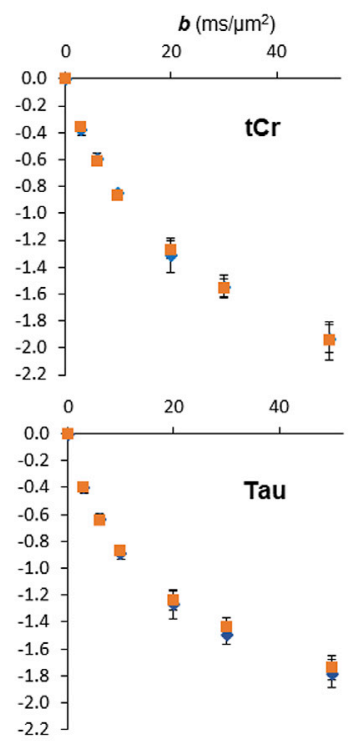

Fig. 2. DW-MRS data at high diffusion-weightings in the CNTF mouse model of reactive astrocytes. (A) Spectra acquired up to high diffusion-weighting (from $b=0.02 \mathrm{~ms} / \mu \mathrm{m}^{2}$ to $50 \mathrm{~ms} / \mu \mathrm{m}^{2}$, at fixed $\mathrm{t}_{\mathrm{d}}=53.2 \mathrm{~ms}$ ) in one control mouse (left) and one CNTF mouse (right). (B) Signal attenuation as a function of diffusion-weighting $b$ for all six intracellular metabolites reliably quantified, in both groups (blue diamond = control group; orange squares $=$ CNTF group). Data points and error bars stand for mean \pm s.d. ( $n=10$ mice per group). Only myoinositol exhibits significantly different diffusion behavior (ANOVA group* $b$ interaction: $\mathrm{p}=0.0048$ ). Unpaired Student's t-tests were run as post hoc tests for each $b$ to assess significantly different data points between groups $\left(*: \mathrm{p}<0.05,{ }^{* *}: \mathrm{p}<0.01,{ }^{* * *}\right.$ : $\mathrm{p}<0.001$, after Bonferroni correction). (Glu $=$ glutamate, Tau $=$ taurine, tCho $=$ choline compounds, $\mathrm{tCr}=$ total creatine). 


$$
\text { A } \quad t_{d}=253.2 m s
$$

Control
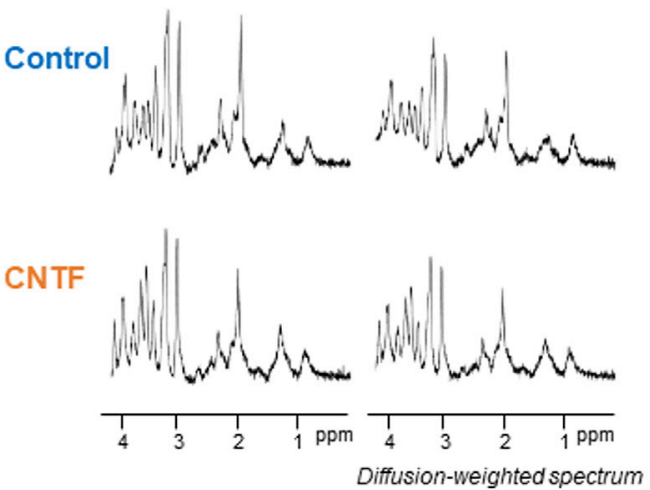

$$
\mathrm{t}_{\mathrm{d}}=1003.2 \mathrm{~ms}
$$
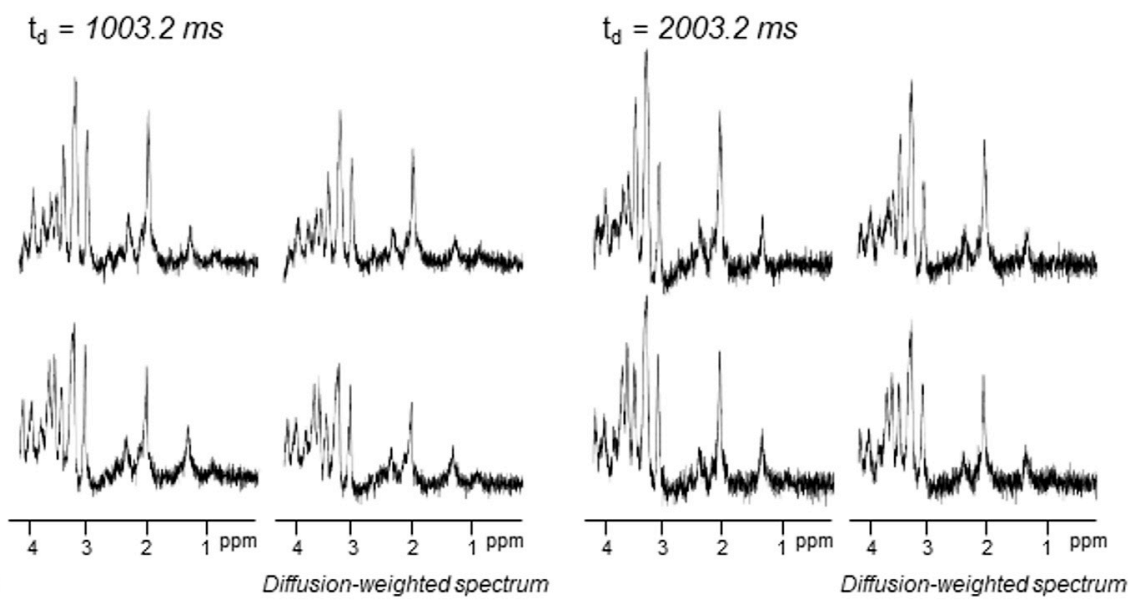

B

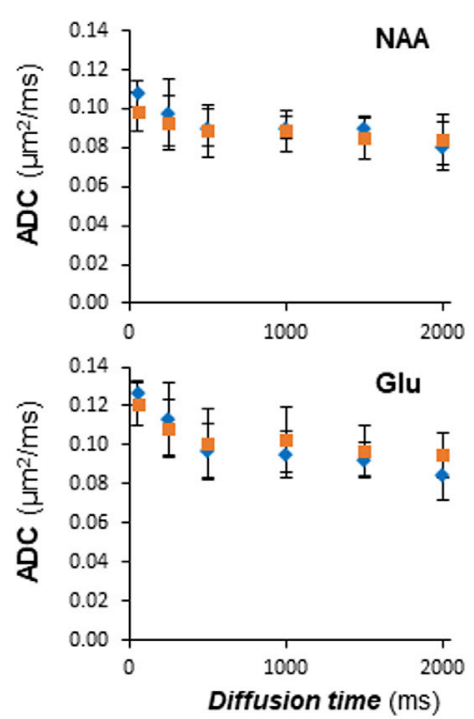

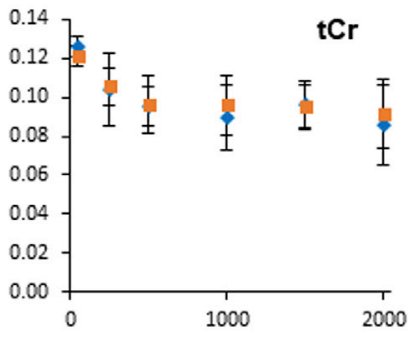

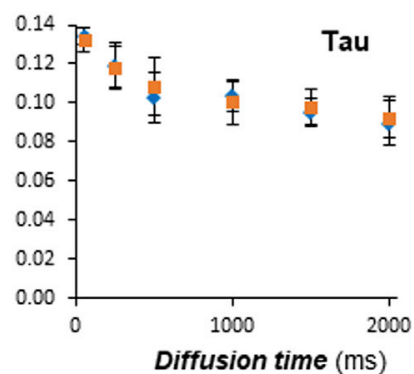

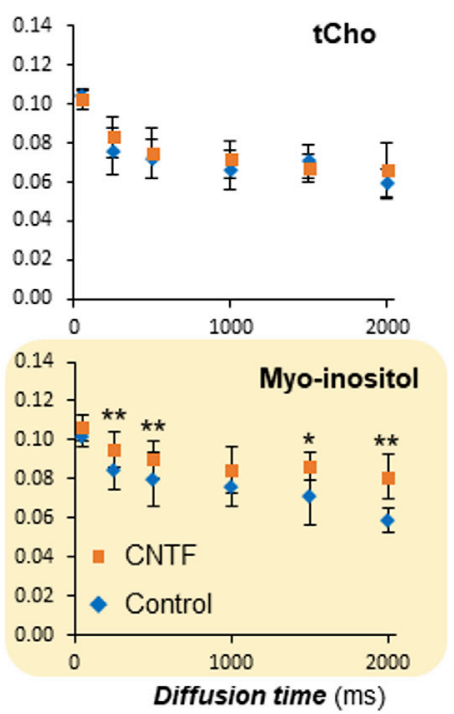

Fig. 3. DW-MRS data at long diffusion times in the CNTF mouse model of reactive astrocytes. (A) Spectra acquired in a control mouse and a CNTF mouse at long diffusion times, without and with diffusion-weighting. Note that the exact $b$-values are given in Methods. (B) Apparent diffusion coefficient (ADC) as a function of the diffusion time $\left(\mathrm{t}_{\mathrm{d}}\right)$ for all six intracellular metabolites reliably quantified, in both groups (blue diamond = control group; orange squares = CNTF group). Data points and error bars stand for mean \pm s.d. ( $n=10$ mice per group). Only myo-inositol exhibits significantly different diffusion behavior (ANOVA group* $t_{d}$ interaction: $\mathrm{p}=0.016)$. Unpaired Student's t-tests were run as post hoc tests for each $\mathrm{t}_{\mathrm{d}}$ to assess significantly different data points between groups $(*: \mathrm{p}<0.05, * *: \mathrm{p}<0.01$, after Bonferroni correction). ( $\mathrm{Glu}=$ glutamate, $\mathrm{Tau}=$ taurine, $\mathrm{tCho}=$ choline compounds, $\mathrm{tCr}=$ total creatine).

Table 2

(A) Fiber and soma radii estimated from confocal microscopy images and from DW-MRS signal attenuation as a function of b for myo-inositol, in control and CNTF mice. The values from ex vivo confocal microscopy ("Real") represent the average values over the population of 20 cells reconstructed from confocal microscopy images (s.d. in brackets). The values from in vivo DW-MRS ("Synthetic") represent the estimated values by fitting equation [3] to the experimental myo-inositol high-b data, with s.d. being evaluated using a Monte Carlo (MC) approach $(n=2500$ draws). Statistical difference between control and CNTF values was assessed by permutation analysis of the parameter distribution estimated from the 2,500 MC draws using unpaired two-tailed $t$-test with Bonferroni correction (** $=\mathrm{p}<0.01 ; * * *=\mathrm{p}<0.001)$. (B) Mean and s.d. of the long-range morphometric statistics ( $\mathrm{L}_{\text {segment }}$ and Branch Order) estimated on 20 cells, reconstructed either from confocal microscopy images ("real") or when fitting the ADC time-dependency of myo-inositol with the computational framework described in the text ("synthetic"). Statistical difference between control and CNTF values was assessed by permutation analysis of the parameter distribution estimated from the $2500 \mathrm{MC}$ draws using unpaired two-tailed $t$-test with Bonferroni

\begin{tabular}{|c|c|c|c|c|c|c|c|}
\hline A & & $\mathrm{R}_{\text {soma }}(\mu \mathrm{m})$ & Variation (\%) & $\mathrm{R}_{\text {fiber }}(\mu \mathrm{m})$ & Variation (\%) & $\mathrm{D}_{\text {intra }}\left(\mu \mathrm{m}^{2} / \mathrm{ms}\right)$ & Variation (\%) \\
\hline \multirow[t]{2}{*}{ Real } & Control & $3.7( \pm 1.2)$ & $+29[* *]$ & $0.50( \pm 0.08)$ & $+20[* *]$ & n.a. & n.a. \\
\hline & CNTF & $4.9( \pm 0.9)$ & & $0.60( \pm 0.13)$ & & n.a. & \\
\hline \multirow{2}{*}{ Synthetic (from Ins high-b) } & Control & $3.80( \pm 0.06)$ & $+23[* * *]$ & $0.58( \pm 0.02)$ & $+47[* * *]$ & $0.375( \pm 0.006)$ & -1 [n.s.] \\
\hline & CNTF & $4.66( \pm 0.07)$ & & $0.85( \pm 0.02)$ & & $0.372( \pm 0.005)$ & \\
\hline $\mathrm{B}$ & & $\mathrm{L}_{\text {segment }}(\mu \mathrm{m})$ & Variation (\%) & Branch Order & Variation (\%) & $\mathrm{D}_{\text {intra }}\left(\mu \mathrm{m}^{2} / \mathrm{ms}\right)$ & Variation (\%) \\
\hline \multirow[t]{2}{*}{ Real } & Control & $15( \pm 5)$ & $+67[* * *]$ & $6( \pm 4)$ & -33 [n.s.] & n.a. & n.a. \\
\hline & CNTF & $25( \pm 7)$ & & $4( \pm 2)$ & & n.a. & \\
\hline \multirow[t]{2}{*}{ Synthetic (from Ins long- $t_{d}$ ) } & Control & $29( \pm 10)$ & $+103[* * *]$ & $6( \pm 5)$ & -17 [n.s.] & $0.376( \pm 0.008)$ & -2 [n.s.] \\
\hline & CNTF & $59( \pm 10)$ & & $5( \pm 5)$ & & $0.369( \pm 0.005)$ & \\
\hline
\end{tabular}
correction $(* * * * \mathrm{p}<0.001)$. 


\subsection{Diffusion modeling allows quantifying structural alterations of myo- inositol diffusion compartment}

We then attempted to model myo-inositol diffusion data to quantitatively evaluate structural alterations of myo-inositol compartment. High diffusion-weighting data were analyzed using analytical models of diffusion in randomly oriented cylinders (Palombo et al., 2017a), slightly modified to also account for diffusion in spherical soma representing $\sim 20 \%$ of the total cell volume, as recently proposed in (Palombo et al., 2018a; 2018b) and summarized in Methods. We extracted fiber radii of $0.58 \pm 0.02 \mu \mathrm{m}$ for the control group versus $0.85 \pm 0.02 \mu \mathrm{m}$ for the CNTF group $(+47 \%)$, and soma radii of $3.80 \pm 0.06 \mu \mathrm{m}$ for the control group versus $4.66 \pm 0.07 \mu \mathrm{m}$ for the CNTF group $(+23 \%)$ (Table $2 \mathrm{~A}$ and Figure S2 A).

Long- $t_{\mathrm{d}}$ myo-inositol data were analyzed using a machine learning approach (Nedjati-Gilani et al., 2017), elaborating upon the framework of numerical simulations in branched structures as we introduced recently (Palombo et al., 2016). Data modeling yields a preserved Branch Order in CNTF, but an increased length of fiber segments between each embranchment, from $29 \pm 3 \mu \mathrm{m}$ to $59 \pm 6 \mu \mathrm{m}(+103 \%)$, resulting in overall increased fiber length (Table 2B).

\subsection{Ex vivo microscopy confirms astrocytic hypertrophy in line with alterations of myo-inositol diffusion compartment}

Examples of astrocytes as imaged with confocal laser scanning microscopy are shown on Fig. 4. On reconstructed GFP-expressing astrocytes, we measured fiber and soma radii of respectively $0.50 \pm 0.08 \mu \mathrm{m}$ and $3.7 \pm 1.2 \mu \mathrm{m}$ for the control group versus $0.60 \pm 0.13 \mu \mathrm{m}$ and $4.9 \pm 0.9 \mu \mathrm{m}$ for the CNTF group $(+20 \%$ and $+32 \%)$. These data confirm thicker astrocytic processes and bigger soma for reactive astrocytes, and are in very good quantitative agreement with values extracted from modeling high-b myo-inositol data (Table $2 \mathrm{~A}$ ).

A
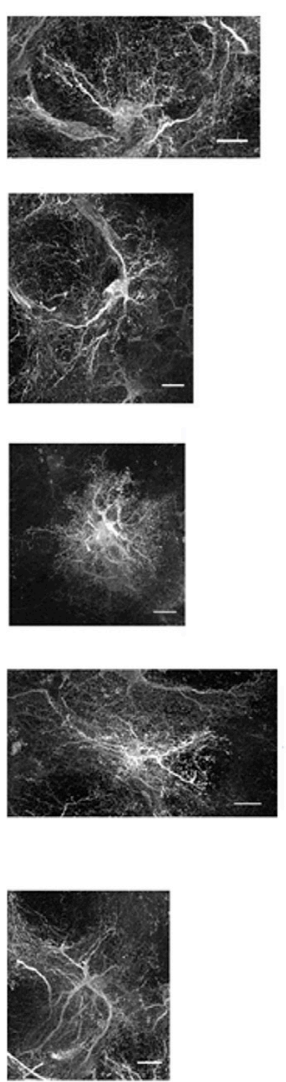
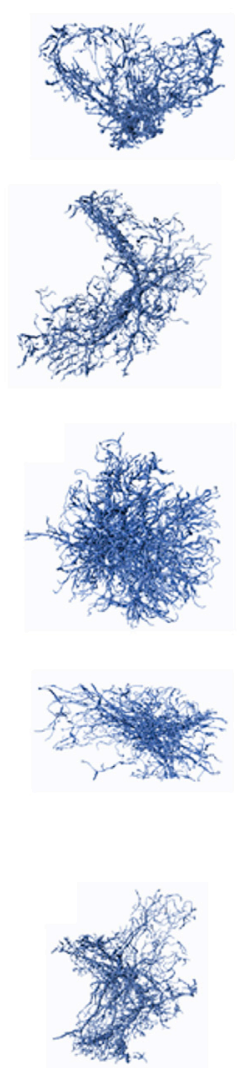

B
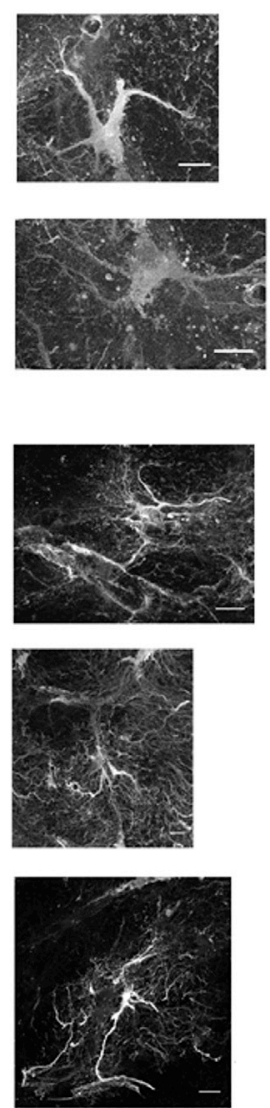

For relevant comparison between long-range morphologies measured from confocal microscopy and from the long- $t_{d}$ myo-inositol data modeling, the structure of astrocytes obtained by confocal microscopy was simplified, as briefly explained in the Methods and detailed in the SI appendix. In the end, after simplification, we found that branching complexity did not change significantly, but we measured increased segment length between embranchments, from $15 \pm 5 \mu \mathrm{m}$ to $25 \pm 7 \mu \mathrm{m}$ $(+67 \%)$. These data confirm longer astrocytic processes for reactive astrocytes, in agreement with the increased length estimated from modeling long- $\mathrm{t}_{\mathrm{d}}$ myo-inositol data (Table $2 \mathrm{~B}$, Fig. S3 for quantitative comparison, and Fig. 5 for visualization). It appears that estimates of $\mathrm{L}_{\text {segment }}$ in synthetic cells are about twice larger than real ones. However, despite that discrepancy in terms of absolute length, the overall agreement between synthetic and real astrocytes remains striking: $\mathrm{L}_{\text {segment }}$ statistically increases in the CNTF group as compared to the control group, while Branch Order tends to decrease, even if not significantly.

To conclude this part, the fact that histology-assessed "ground truth" astrocytic morphology and its alterations are well reflected by myoinositol's diffusion brings strong quantitative evidence that myo-inositol is predominantly localized into astrocytes.

\subsection{Massive variations of lactate diffusion suggest an alteration of lactate cellular compartmentation}

Besides the intracellular metabolites, we also report the signal attenuation of lactate, which is present both in the intracellular and extracellular compartments. As can be noted from Fig. 6B, its signal attenuation as a function of $b$ in both conditions cannot be compared to other metabolites (very strong signal attenuation between $b=0.05 \mathrm{~ms}$ / $\mu \mathrm{m}^{2}$ and $b=3.050 \mathrm{~ms} / \mu \mathrm{m}^{2}$ ), as expected from the partial contribution of the extracellular lactate (Hillered et al., 1989; Nilsson and Ponten, 1990) presumably resulting in faster diffusion and hence steeper signal
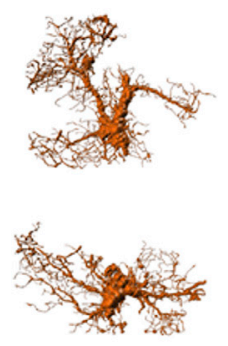

Fig. 4. (A) Examples of real astrocytes from control mice. (A-Left) Confocal images maximum intensity projection of different GFP-labeled striatal astrocytes of mice in which neurons secreted $\beta$-Galactosidase as control; in all cases, scale bars correspond to $10 \mu \mathrm{m}$. (A-Right) Reconstructions based on the confocal images of each selected astrocytes. (B) Examples of real astrocytes from CNTF mice. (B-Left) Confocal images maximum intensity projection of different GFPlabeled striatal astrocytes of mice in which neurons secreted CNTF; in all cases, scale bars correspond to $10 \mu \mathrm{m}$. (B-Right) Reconstructions based on the confocal images of each selected astrocyte. 


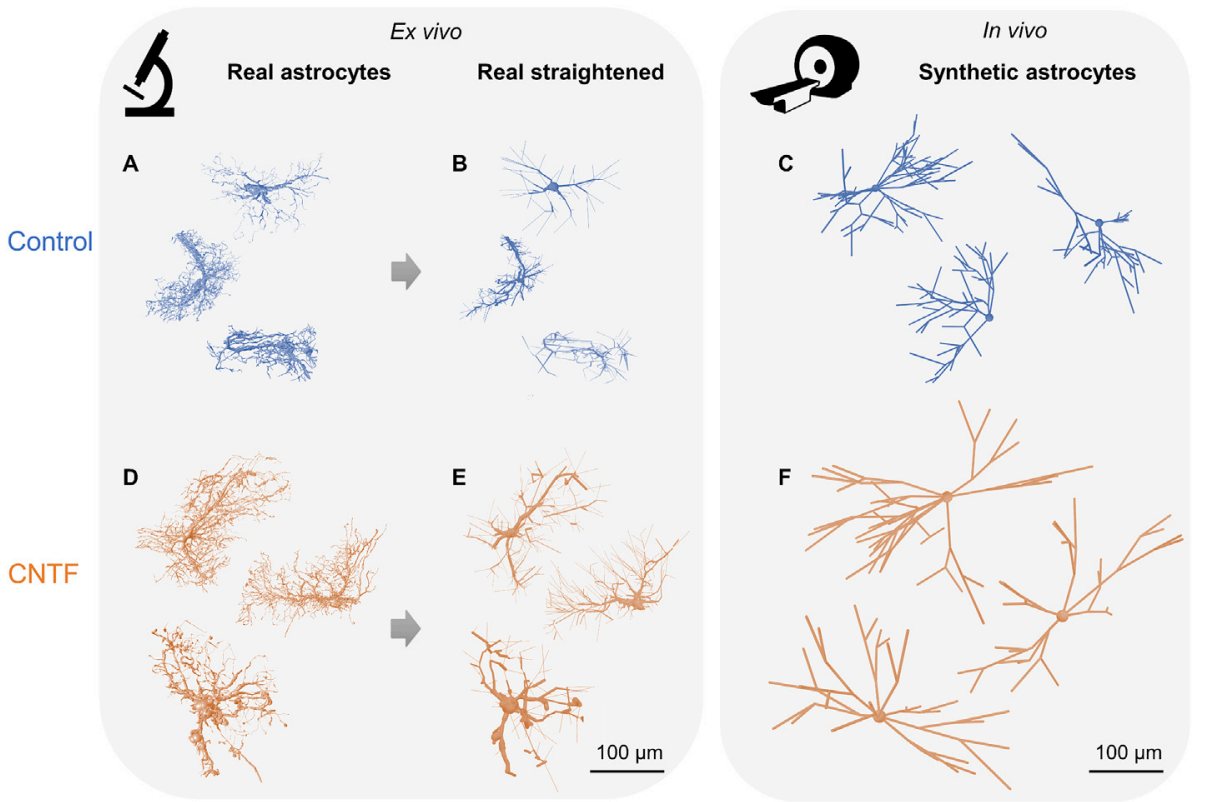

Fig. 5. Comparison of some astrocytes obtained from direct ex vivo measurement by confocal laser scanning microscopy ("real" astrocytes) in control (A) and CNTF (D) mice; and from modeling of in vivo myoinositol DW-MRS data ("synthetic" astrocytes) in control (C) and CNTF (F) mice. (B) and (E) represent typical astrocytes reconstructed from confocal microscopy after simplification of their structure ("real straightened" astrocytes, see Methods and SI appendix), from which we measure morphological parameters for quantitative comparison with synthetic astrocytes. In both real straightened and synthetic astrocytes, increased soma radius, fiber radius and fiber length are measured in CNTF (see main text and Table 2 for parameter values). To generate the representations for synthetic astrocytes in (C) and (F), some cell-graphs as extracted from long- $t_{d}$ data were "thickened" by conferring some non-zero radius to their fibers and soma, corresponding to the best radii extracted from high- $b$ data for each group.
A

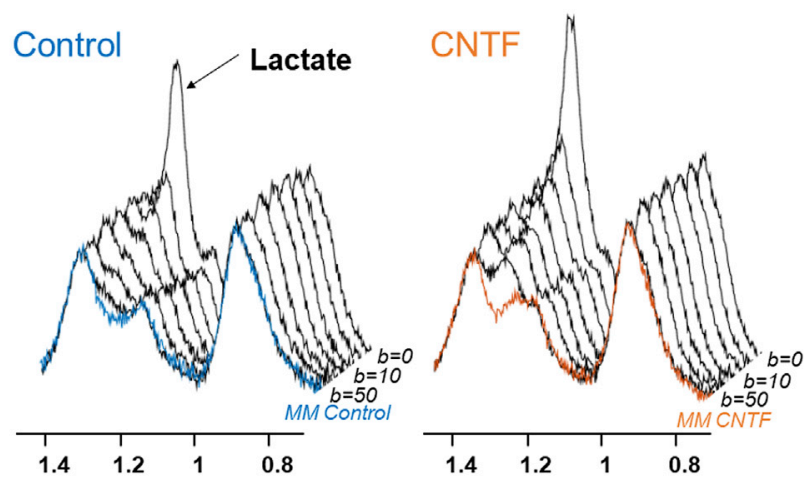

D

\section{Control group}

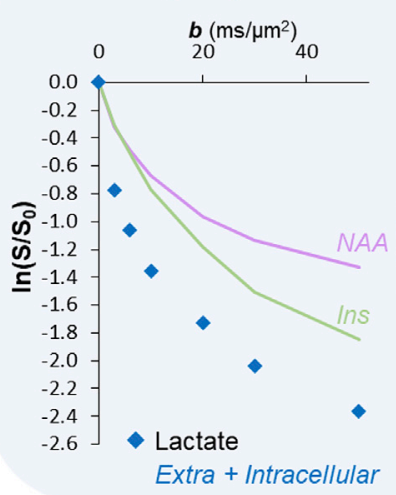

B

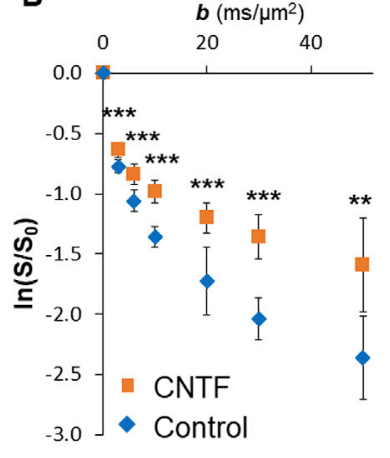

E

\section{CNTF group}

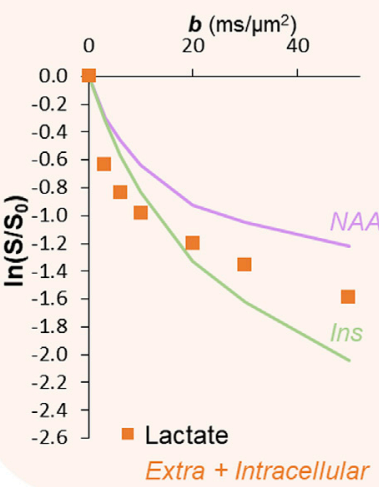

C

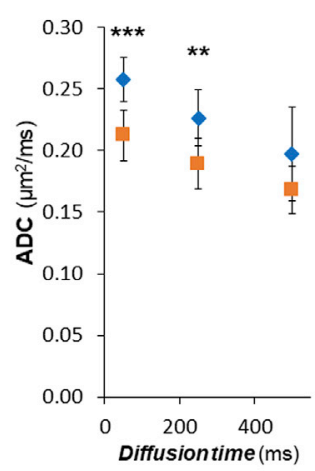

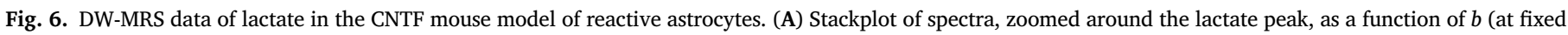

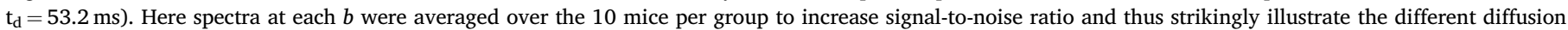

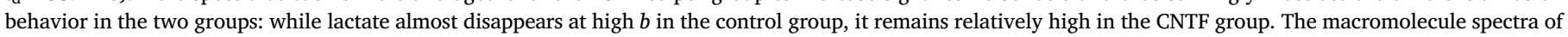

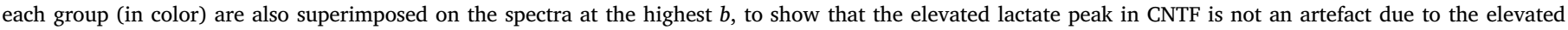

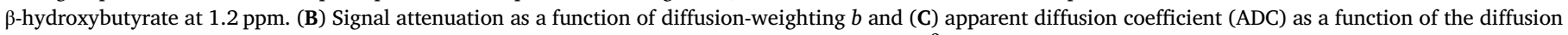

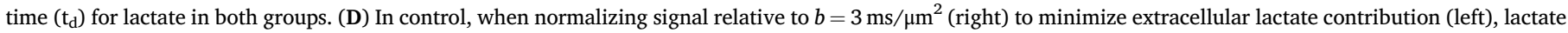

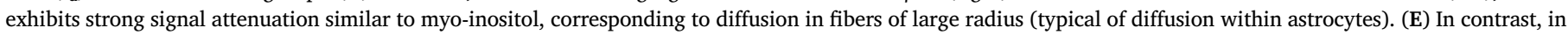

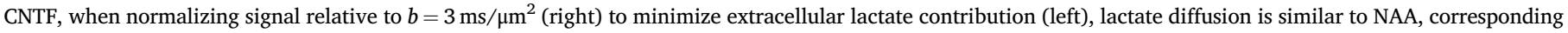
to diffusion in fibers of small radius (typical of diffusion within neurons). 
attenuation at low $b$-values (Pfeuffer et al., 2000). However, it is worth noting that lactate signal attenuation differs drastically between both groups, suggesting that lactate is experiencing more restricted diffusion in the CNTF group.

For the sake of consistency, we also report the ADC measured for lactate (Fig. 6C). As pointed out from Fig. 6B, the fast signal attenuation of lactate in the low $b$-values range is weighted by its significant extracellular component. Hence the lower ADC up to $t_{d}=503.2 \mathrm{~ms}$ in the CNTF group might reflect the fact that the extracellular fraction of lactate is lower in this group as compared to the control group. Data at longer $t_{d}$ are too noisy to be reliable (increase of CRLBs) and are therefore not reported.

Interpreting lactate diffusion is a delicate matter. Firstly, the anesthetic has a strong influence on lactate concentration in rodent brain (Boretius et al., 2013), meaning that the metabolic state is not exactly basal. Since conditions of anesthesia were identical in both groups, the comparison still holds, keeping in mind that the conclusions might depend on the anesthetic. Secondly, unlike other metabolites, lactate has a significant extracellular fraction. However, considering that the extracellular contribution mostly causes a drop of signal attenuation at low diffusion-weightings, the signal attenuation at high-diffusion weightings should be mainly driven by its intracellular component. For visual inspection, Fig. 6D-E shows signal attenuation of NAA, Ins and lactate in the CNTF (Fig. 6E) and control group (Fig. 6D) normalized by signal acquired at $b=3.050 \mathrm{~ms} / \mu \mathrm{m}^{2}$ to tentatively minimize the extracellular contribution of lactate. Intracellular lactate diffusion in the control group appears then very similar to myo-inositol, suggesting intracellular lactate is predominantly compartmentalized in astrocytes. In contrast, in the CNTF group, intracellular lactate diffusion is very similar to NAA (which is generally considered as a highly specific neuronal metabolite), suggesting that intracellular lactate is now predominantly found in neurons.

\section{Discussion}

\subsection{Myo-inositol diffusion reflects astrocytic morphology and its alterations}

The primary finding of this work is the identification of myo-inositol as the intracellular metabolite whose diffusion is the most sensitive and specific to astrocytic morphological changes. Actually, among intracellular metabolites, significant diffusion alterations could only be detected for myo-inositol.

Although its role in the brain is still poorly understood, myo-inositol has been proposed as an astrocytic osmolite (Isaacks et al., 1999; Thurston et al., 1989; Strange et al., 1994), and increased myo-inositol concentration is generally associated with astrogliosis. Our results show a strong increase in myo-inositol concentration in the CNTF group. In the light of this work we can, at least to some extent, ascribe this increased myo-inositol content to the increased volume fraction occupied by astrocytes in the volume of interest, due to astrocytic hypertrophy. However, some explanations related to energy metabolism might also be invoked (see next section).

Because myo-inositol diffusion is altered in the context of hypertrophic astrocytes, it is very tempting to consider myo-inositol compartment shape as a proxy for astrocytic morphology. It is worth mentioning that metabolites' dynamics within the intracellular space are very complex. Indeed, other mechanisms different from diffusion may occur at the same time. For example, metabolites may be involved in active transport and flow; they may be confined in sub-compartments like small organelles and mitochondria or be involved in electro-chemical interactions with macromolecules. However, the time dependence of metabolites ADC may provide insights about the contribution of these mechanisms relative to the diffusion process. If active transport and coherent flow of metabolites were not negligible compared to diffusion, then the measured ADC should increase as a function of the diffusion time. Since we did not observe any significant increase of the ADC of any metabolite at increasing diffusion time, we can conclude that these phenomena are negligible compared to diffusion. On the other hand, if metabolites were confined in small organelles or mitochondria the ADC should approach zero at the longest $t_{d}$ values investigated here. On the contrary, we measured a fairly stable $\mathrm{ADC}$ as a function of the diffusion time, suggesting that the fraction of metabolites fully confined in small sub-compartments is negligible compared to the fraction of metabolites able to diffuse within the cellular cytoplasm. Finally, our measure is only sensitive to bulk metabolites: any metabolite bound to a protein vanishes in the macromolecules baseline. Therefore, based on pure data observation, these qualitative remarks highlight again that diffusion of metabolites mostly depend on the cellular structure constraining them, in agreement with observations in previous works (Marchadour et al., 2012; Najac et al., 2014; Ligneul et al., 2017; Palombo et al., 2016). It turns out that soma radii and process lengths and radii estimated by modeling myo-inositol diffusion are indeed very consistent with actual astrocytic morphology: the agreement between parameters extracted from DW-MRS modeling and confocal microscopy is in general excellent, except for the absolute length of segments. The fact that DW-MRS and confocal microscopy do not yield identical segment length might have different explanations. On one hand, diffusion models are simplified approximations of reality, and as such cannot be anticipated to perfectly reflect cell morphology. In the same vein, the astrocytic specificity of myo-inositol is certainly not perfect, i.e. myo-inositol diffusion might to some extent also reflect the morphology of other cell types (such as neurons, which exhibit longer projections than astrocytes) where myo-inositol might be present at detectable levels. On the other hand, confocal microscopy samples only a few cells which might not necessarily represent the whole cell population within the spectroscopic volume of interest. Furthermore it cannot access the extremity of astrocytic processes if they go below optical resolution, thus presumably underestimating the length of the thinnest segments. Finally, confocal microscopy is an ex vivo technique requiring tissue preparation which usually results in some shrinkage and cutting of histological sections; since in the present study only full astrocytes were analyzed, the longest segments/processes extending beyond slice thickness may have been systematically excluded, resulting in underestimated length. Whatever the origin of this discrepancy, myo-inositol diffusion still appears to be very sensitive to astrocyte reactivity, consistently with astrocytic hypertrophy.

The fact that other intracellular metabolites do not exhibit significant diffusion variations, in contrast to myo-inositol, is a strong indication that their astrocytic fraction is small and does not dominate their MRS signal. While this may not be surprising for neuronal metabolites such as NAA and glutamate, or even for non-specific metabolites such as total creatine or taurine, for which it can be conceived that the neuronal contribution dominates, this is rather conflicting with some previous reports of choline compounds being highly abundant in astrocytes cells. A possible explanation we can speculate on might be that astrocytes come in two distinct sub-populations, one being enriched with myo-inositol and becoming hypertrophic, the other being enriched with choline compounds and preserving its morphology.

\subsection{Alterations of energy metabolism in the CNTF model of reactive astrocytes}

The change in signal attenuation measured for lactate, mirroring the signal attenuation of NAA in the CNTF group, likely reflects some major remodeling of lactate cellular compartmentation. In the control group, DW-MRS suggests that intracellular lactate is predominantly in astrocytes, not in neurons. This is in line with a recent study using the Laconic FRET sensor in the mouse brain (Mächler et al., 2016), showing that astrocytes maintain significantly higher lactate levels than neurons, and consistent with the astrocyte-to-neuron lactate shuttle (ANLS) hypothesis (Pellerin and Magistretti, 1994), which requires a larger concentration of lactate in astrocytes as compared to neurons. In contrast, intracellular lactate appears to be predominantly in neurons in the CNTF group. This rules out the possibility for ANLS in the CNTF group. Incidentally, the disruption of ANLS is consistent with the diminished extracellular 

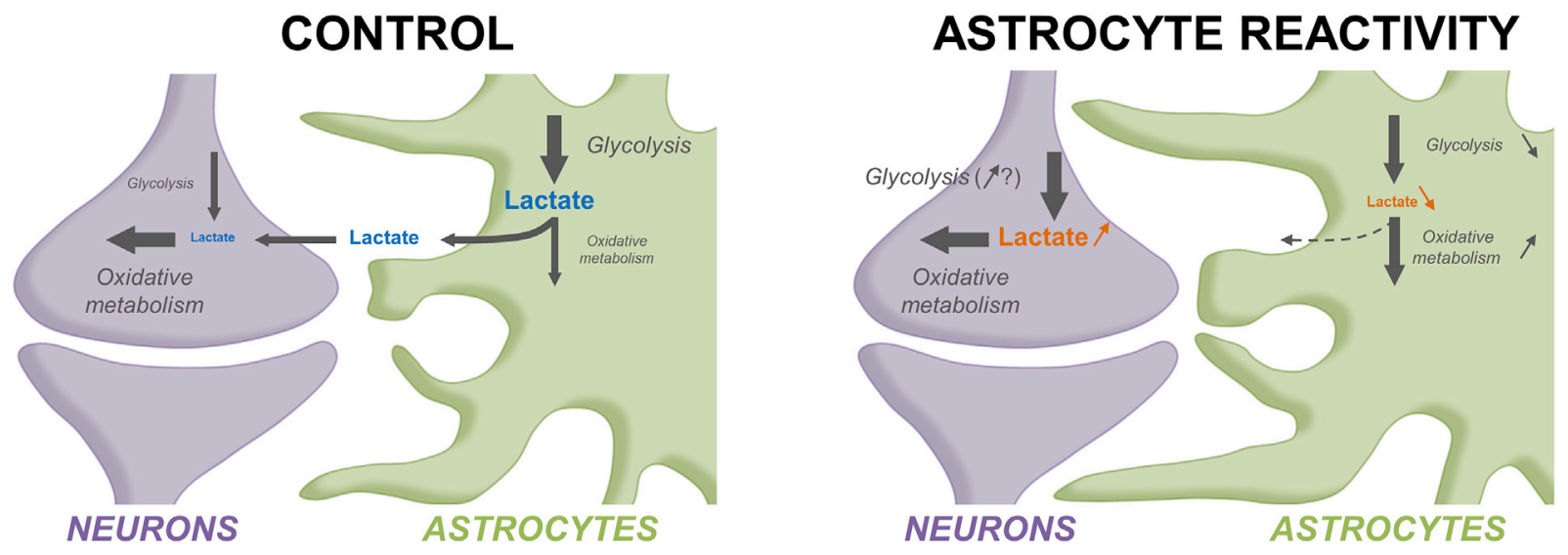

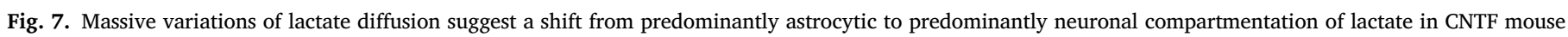
model of reactive astrocytes, leading to disruption of the astrocyte-to-neuron lactate shuttle.

fraction suggested from lower lactate ADC in the CNTF group (Fig. 6C). How does this remodeling of lactate compartmentation, as suggested by DW-MRS and recapitulated in Fig. 7, integrate with other metabolic features of CNTF reactive astrocytes?

Increased oxidative metabolism in reactive astrocytes has been shown to be associated notably with an overexpression of $\beta$-hydroxybutyrate dehydrogenase $(\mathrm{BDH})$, providing an alternative source of acetyl-CoA by metabolizing ketone bodies (Escartin et al., 2007). This might explain the presence of the increased peak at $1.2 \mathrm{ppm}$ on the macromolecule spectrum of the CNTF group, presumably corresponding to more $\beta$-hydroxybutyrate interacting with BDH. Moreover, decreased glucose uptake and lactate dehydrogenase (LDH) activity have also been reported, pointing towards an overall decreased glycolytic activity in CNTF astrocytes. Interestingly, this is in line with the massive increase of myo-inositol concentration in the CNTF group. Indeed, myo-inositol is found in food, but certain part of the body with higher basal concentrations of myo-inositol can also produce their own myo-inositol, like the kidney and the brain, associated with high metabolism of inositol phospholipids (Hauser and Finelli, 1963). This process involves the transformation of D-Glucose-6P (directly from glucose) in D-myo-inositol-3P, that dephosphorylates into myo-inositol (Fisher et al., 2002), under the action of myo-inositol-3-phosphatesynthase (INO1) and inositolmonophosphatase (IMPase). Moreover, the uptake of glucose is stable in CNTF reactive astrocytes. If the myo-inositol uptake from the blood is stable (implying that SMIT1 and SGLT6 are not upregulated), the cell must produce more myo-inositol, meaning that less D-Glucose-6P enters the glycolysis, concomitantly explaining downregulation of the glycolysis in astrocytes, and the observed decreased production of lactate in astrocytes. In that context, all pyruvate/lactate produced by astrocytes is likely to be locally consumed to feed increased oxidative metabolism, rather than accumulating in astrocytes; to supplement the deficiency of astrocytic lactate production and the resulting disruption of ANLS, neurons may adjust their metabolism and synthesize more lactate, thus resulting in elevated lactate concentrations in neurons.

Although demanding further investigation, these data and their interpretation suggest that DW-MRS may allow non-invasively probing cellular compartmentation of lactate. This would open unprecedented possibilities to study lactate metabolism and astrocyte-neuron interactions in various contexts, such as brain activity (Pellerin and Magistretti, 1994), synaptic plasticity and long-term memory (Suzuki et al., 2011; Newman et al., 2011; Pellerin and Magistretti, 2012; Magistretti, 2014), and Alzheimer's disease (Demetrius et al., 2014; Harris et al., 2016).

\section{Conclusion}

In conclusion, specific alterations of intracellular metabolite diffusion could be measured and related, for the first time and quantitatively, to cell-specific morphological alteration as independently assessed by microscopy. More specifically, this work establishes DW-MRS as a unique tool to non-invasively monitor astrocytic structural alterations via specific variations of myo-inositol diffusion. A complementary step in the future would be to detect specific variations of the diffusion of neuronal metabolites (NAA, Glu) in a context of altered neuronal morphology. Beyond cell structure, this work also suggests that DW-MRS may yield new insights into lactate cellular compartmentation, which is tightly related to the important but still controversial notion of astrocyte-to-neuron lactate shuttling (Suzuki et al., 2011; Diaz-Garcia et al., 2017). Since no fundamental barrier prevents implementing similar approaches on clinical MRI scanners, the strategy developed here may provide unprecedented access to astrocyte reactivity in the Human brain, especially now that stronger clinical gradients are becoming available (e.g. up to $300 \mathrm{mT} / \mathrm{m}$ along each axis for the Human Connectome Project (Jones et al., 2018)). This could lead to better understanding of the role and status of astrocytes in the healthy and diseased Human brain, and allow monitoring the effect of treatments modulating astrocyte reactivity. Although the present implementation only deals with single-volume detection, recent and promising developments in diffusion-weighted spectroscopic imaging (Ercan et al., 2014; Bito et al., 2015; Fotso et al., 2017) let us foresee the possibility to quantitatively map myo-inositol diffusion, opening the way towards cell-specific microstructure imaging throughout the Human brain.

\section{Competing interest}

The authors have no conflict of interest to disclose.

\section{Authors contribution}

Conceptualization: J.V., C.L., M.P., G.B., C.E. Methodology: J.V, C.L., M.P., E.H.G., M.C.S, J.F. Software: J.V., M.P., C.L., E.H.G Investigation C.L., M.P., E.H.G. Writing: J.V, C.L., M.P., E.H.G. Resources: P.H., E.B. Supervision: J.V. Funding Acquisition: J.V.

\section{Acknowledgements}

This research was supported by the European Research Council, European Union (grant agreement 336331 - INCELL project, awarded to J.V.). M.P. acknowledges support from EPSRC, United Kingdom grant EP/N018702/1. The 11.7 T MRI scanner and the confocal microscope were funded by a grant from "Investissements d'Avenir - ANR-11-INBS0011 - NeurATRIS: A Translational Research Infrastructure for Biotherapies in Neurosciences". 
Appendix A. Supplementary data

Supplementary data to this article can be found online at https://doi. org/10.1016/j.neuroimage.2019.02.046.

\section{References}

Alexander, D.A., Dyrby, T.B., Nilsson, M., Zhang, H., 2017. Imaging brain microstructure with diffusion MRI: practicality and applications. NMR Biomed. https://doi.org/ $10.1002 / \mathrm{nbm} .3841$.

Andersson, J.L., Sotiropoulos, S.N., 2016. An integrated approach to correction for offresonance effects and subject movement in diffusion MR imaging. Neuroimage 125, 1063-1078.

Araque, A., et al., 2014. Gliotransmitters travel in time and space. Neuron 81 (4), 728-739.

Ben Haim, L., Carrillo-de Sauvage, M.-A., Ceyzériat, K., Escartin, C., 2015. Elusive roles for reactive astrocytes in neurodegenerative diseases. Front. Cell. Neurosci. 9, 278

Bito, Y., et al., 2015. Diffusion-weighted line-scan echo-planar spectroscopic imaging technique to reduce motion artifacts in metabolite diffusion imaging. Magn. Reson. Med. Sci. 14 (1), 43-50.

Basser, P.J., Mattiello, J., LeBihan, D., 1994. Estimation of the effective self-diffusion tensor from the NMR spin echo. J. Magn. Reson. B 103, 247-254.

Basser, P., Pierpaoli, C., 1996. Microstructural and physiological features of tissues elucidated by quantitative diffusion-tensor MRI. J. Magn. Reson. 111, 209-219.

Boretius, S., Tammer, R., Michaelis, T., Brockmöller, J., Frahm, J., 2013. Halogenated volatile anesthetics alter brain metabolism as revealed by proton magnetic resonance spectroscopy of mice in vivo. Neuroimage 69, 244-255.

Carrillo-de Sauvage, M.A., et al., 2015. The neuroprotective agent CNTF decreases neuronal metabolites in the rat striatum: an in vivo multimodal magnetic resonance imaging study. J. Cerebr. Blood Flow Metabol. 35 (6), 917-921.

Chvatal, A., Anderova, M., Kirchhoff, F., 2007. Three-dimensional confocal morphometry - a new approach for studying dynamic changes in cell morphology in brain slices. J. Anat. 210 (6), 671-683.

Colin, A., et al., 2009. Engineered lentiviral vector targeting astrocytes in vivo. Glia 57 (6), 667-679.

Cuntz, H., Forstner, F., Borst, A., Hausser, M., 2010. One rule to grow them all: a general theory of neuronal branching and its practical application. PLoS Comput. Biol. 6 (8),

Demetrius, L.A., Magistretti, P.J., Pellerin, L., 2014. Alzheimer's disease: the amyloid hypothesis and the Inverse Warburg effect. Front. Physiol. 5, 522.

Diaz-Garcia, C.M., et al., 2017. Neuronal stimulation triggers neuronal glycolysis and not lactate uptake. Cell Metabol. 26 (2), 361-374.

Dossi, E., Vasile, F., Rouach, N., 2018. Human astrocytes in the diseased brain. Brain Res. Bull. 136, 139-156.

Ercan, A.E., Techawiboonwong, A., Versluis, M.J., Webb, A.G., Ronen, I., 2014. Diffusionweighted chemical shift imaging of human brain metabolites at 7T. Magn. Reson. Med. 73 (6), 2053-2061.

Ercan, E., et al., 2016. Glial and axonal changes in systemic lupus erythematosus measured with diffusion of intracellular metabolites. Brain 139 (Pt 5), 1447-1457.

Escartin, C., et al., 2006. Ciliary neurotrophic factor activates astrocytes, redistributes their glutamate transporters GLAST and GLT-1 to raft microdomains, and improves glutamate handling in vivo. J. Neurosci. 26 (22), 5978-5989.

Escartin, C., et al., 2007. Activation of astrocytes by CNTF induces metabolic plasticity and increases resistance to metabolic insults. J. Neurosci. 27 (27), 7094-7104.

Fisher, S.K., Novak, J.E., Agranoff, B.W., 2002. Inositol and higher inositol phosphates in neural tissues : homeostasis, metabolism and functional significance. J. Neurochem $82,736-754$.

Fotso, K., et al., 2017. Diffusion tensor spectroscopic imaging of the human brain in children and adults. Magn. Reson. Med. 78 (4), 1246-1256.

Garyfallidis, E., Brett, M., Amirbekian, B., Rokem, A., van der Walt, S., Descoteaux, M., Nimmo-Smith, I., Contributors, Dipy, 2014. DIPY, a library for the analysis of diffusion MRI data. Front. Neuroinf. 8 (8).

Gill, S.S., et al., 1989. Brain metabolites as 1H NMR markers of neuronal and glial disorders. NMR Biomed. 2 (5-6), 196-200.

Griffin, J.L., Bollard, M., Nicholson, J.K., Bhakoo, K., 2002. Spectral profiles of cultured neuronal and glial cells derived from HRMAS 1H NMR spectroscopy. NMR Biomed. 15, 375-384.

Harris, R.A., et al., 2016. Aerobic glycolysis in the frontal cortex correlates with memory performance in wild-type mice but not the APP/PS1 mouse model of cerebral amyloidosis. J. Neurosci. 36 (6), 1871-1878.

Hauser, G., Finelli, V.N., 1963. The biosynthesis of free and phosphatide myo-inositol from glucose by mammalian tissue slices. J. Biol. Chem. 238, 3224-3228.

Haydon, P.G., Nedergaard, M., 2015. How do astrocytes participate in neural plasticity? Cold Spring Harb. Perspect. Biol. 7, a020438.

Hillered, L., Hallstrom, A., Segersvard, S., Persson, L., Vngerstedt, V., 1989. Dynamics of extracellular metabolites in the striatum after middle cerebral artery occlusion in the rat monitored by intracerebral microdialysis. J. Cerebr. Blood Flow Metabol. 9, 607-616.

Isaacks, R.E., Bender, A.S., Kim, C.Y., Shi, Y.F., Norenberg, M.D., 1999. Effect of osmolality and anion channel inhibitors on myo-inositol efflux in cultured astrocytes. J. Neurosci. Res. 57 (6), 866-871.

Jensen, J.H., Helpern, J.A., Ramani, A., Lu, H., Kaczynski, K., 2005. Diffusional kurtosis imaging: the quantification of non_Gaussian water diffusion by means of magnetic resonance imaging. Magn. Reson. Med. 53, 1432-1440.
Jones, D.K., Alexander, D.C., Bowtell, R., Cercignani, M., Dell'Acqua, F., McHugh, D.J., Miller, K.L., Palombo, M., Parker, G.J., Rudrapatna, U.S., Tax, C.M.W., 2018. Microstructural imaging of the human brain with a 'super-scanner': 10 key advantages of ultra-strong gradients for diffusion MRI. Neuroimage 182, 8-38.

Kellner, E., Dhital, B., Kiselev, V.G., Reisert, M., 2016. Gibbs-ringing artifact removal based on local subvoxel-shifts. Magn. Reson. Med. 76 (5), 1574-1581.

Liddelow, S.A., Barres, B.A., 2017. Reactive astrocytes: production, function, and therapeutic potential. Immunity 46 (6), 957-967.

Ligneul, C., Palombo, M., Valette, J., 2017. Metabolite diffusion up to very high b in the mouse brain in vivo: revisiting the potential correlation between relaxation and diffusion properties. Magn. Reson. Med. 77 (4), 1390-1398.

Linse, P., Soderman, O., 1995. The validity of the short-gradient-pulse approximation in Nmr-studies of restricted diffusion - simulations of molecules diffusing between planes, in cylinders and spheres. J. Magn. Reson., Ser. A 116 (1), 77-86.

Mächler, P., et al., 2016. In vivo evidence for a lactate gradient from astrocytes to neurons. Cell Metabol. 23 (1), 94-102.

Magistretti, P.J., 2014. Synaptic plasticity and the Warburg effect. Cell Metabol. 19 (1), 4-5.

Marchadour, C., Brouillet, E., Hantraye, P., Lebon, V., Valette, J., 2012. Anomalous diffusion of brain metabolites evidenced by diffusion-weighted magnetic resonance spectroscopy in vivo. J. Cerebr. Blood Flow Metabol. 32 (12), 2153-2160.

Najac, C., Marchadour, C., Guillermier, M., Houitte, D., Slavov, V., Brouillet, E., Hantraye, P., Lebon, V., Valette, J., 2014. Intracellular metabolites in the primate brain are primarily localized in long fibers rather than in cell bodies, as shown by diffusion-weighted magnetic resonance spectroscopy. Neuroimage 90, 374-380.

Navarrete, M., Araque, A., 2014. The Cajal school and the physiological role of astrocytes: a way of thinking. Front. Neuroanat. 8, 33.

Nedjati-Gilani, G.L., Schneider, T., Hall, M.G., Cawley, N., Hill, I., Ciccarelli, O., Drobnjak, I., Gandini Wheeler-Kingshott, C.A.M., Alexander, D., 2017. Machine learning based compartment models with permeability for white matter microstructure imaging. Neuroimage 150, 119-135.

Newman, L.A., Korol, D.L., Gold, P.E., 2011. Lactate produced by glycogenolysis in astrocytes regulates memory processing. PLoS One 6 (12), e28427.

Nilsson, P., Ponten, U., 1990. Changes in cortical extracellular levels of energy-related metabolites and amino acids following concussive brain injury in rats. J. Cerebr. Blood Flow Metabol. 10, 631-637.

Palombo, M., et al., 2016. New paradigm to assess brain cell morphology by diffusionweighted MR spectroscopy in vivo. Proc. Natl. Acad. Sci. U.S.A. 113 (24), 6671-6676.

Palombo, M., Ligneul, C., Valette, J., 2017a. Modeling diffusion of intracellular metabolites in the mouse brain up to very high diffusion-weighting: diffusion in long fibers (almost) accounts for non-monoexponential attenuation. Magn. Reson. Med. 77 (1), 343-350.

Palombo, M., Ligneul, C., Hernandez-Garzon, E., Valette, J., 2017b. Can we detect the effect of spines and leaflets on the diffusion of brain intracellular metabolites? Neuroimage 182, 283-293.

Palombo, M., Shemesh, N., Ronen, I., Valette, J., 2017c. Insights into brain microstructure from in vivo DW-MRS. Neuroimage 182, 97-116.

Palombo, M., Shemesh, N., Ianus, A., Alexander, D.C., Zhang, H., 2018a. A compartment based model for non-invasive cell body imaging by diffusion MRI. Proc. Int. Soc. Magn. Reson. Med. 27, 6685.

Palombo, M., Shemesh, N., Ianus, A., Alexander, D.C., Zhang, H., 2018b. Abundance of cell bodies can explain the stick model's failure in grey matter at high b-value. Proc. Int. Soc. Magn. Reson. Med. 27, 6170.

Pedregosa, F., Varoquaux, G., Gramfort, A., Michel, V., Thirion, B., Grisel, O., Blondel, M., Prettenhofer, P., Weiss, R., Dubourg, V., Vanderplas, J., Passos, A., Cournapeau, D., Brucher, M., Perrot, M., Duchesnay, E., 2011. Scikit-learn: machine learning in Python. J. Mach. Learn. Res. 12, 2825-2830.

Pellerin, L., Magistretti, P.J., 1994. Glutamate uptake into astrocytes stimulates aerobic glycolysis - a mechanism coupling neuronal-activity to glucose-utilization. Proc. Natl. Acad. Sci. U.S.A. 91 (22), 10625-10629.

Pellerin, L., Magistretti, P.J., 2012. Sweet sixteen for ANLS. J. Cerebr. Blood Flow Metabol. 32 (7), 1152-1166.

Peng, H., Bria, A., Zhou, Z., Iannello, G., Long, F., 2014. Extensible visualization and analysis for multidimensional images using Vaa3D. Nat. Protoc. 9 (1), 193-208.

Pittà, M., Brunel, N., Volterra, A., 2016. Astrocytes: orchestrating synaptic plasticity? Neuroscience $323,43-46$

Pfeuffer, J., Tkac, I., Gruetter, R., 2000. Extracellular-intracellular distribution of glucose and lactate in the rat brain assessed noninvasively by diffusion-weighted $1 \mathrm{H}$ nuclear magnetic resonance spectroscopy in vivo. J. Cerebr. Blood Flow Metabol. 20 (4), $736-746$.

Provencher, S.W., 1993. Estimation of metabolite concentrations from localized in vivo proton NMR spectra. Magn. Reson. Med. 30 (6), 672-679.

Schindelin, J., Arganda-Carreras, I., Frise, E., Kaynig, V., Longair, M., Pietzsch, T., Preibisch, S., Rueden, C., Saalfeld, S., Schmid, B., Tinevez, J.Y., White, D.J., Hartenstein, V., Eliceiri, K., Tomancak, P., Cardona, A., 2012. Fiji: an open-source platform for biological-image analysis. Nat. Methods 9 (7), 676-682.

Scorcioni, R., Polavaram, S., Ascoli, G.A., 2008. L-Measure: a web-accessible tool for the analysis, comparison and search of digital reconstructions of neuronal morphologies. Nat. Protoc. 3 (5), 866-876.

Strange, K., Emma, F., Paredes, A., Morrison, R., 1994. Osmoregulatory changes in myoinositol content and $\mathrm{Na}+$ /myo-inositol cotransport in rat cortical astrocytes. Glia 12 (1), 35-43.

Suzuki, A., et al., 2011. Astrocyte-neuron lactate transport is required for long-term memory formation. Cell 144 (5), 810-823. 
Tabesh, A., Jensen, J.H., Ardekani, B.A., Helpern, J.A., 2011. Estimation of tensors and tensor-derived measures in diffusional kurtosis imaging. Magn. Reson. Med. 65 (3), 823-836.

Thurston, J.H., Sherman, W.R., Hauhart, R.E., Kloepper, R.F., 1989. Myo-inositol: a newly identified nonnitrogenous osmoregulatory molecule in mammalian brain. Pediatr. Res. 26 (5), 482-485.

Tyzack, G.E., et al., 2014. Astrocyte response to motor neuron injury promotes structural synaptic plasticity via STAT3-regulated TSP-1 expression. Nat. Commun. 5, 4294.

Urenjak, J., Williams, S.R., Gadian, D.G., Noble, M., 1993. Proton nuclear magnetic resonance spectroscopy unambiguously identifies different neural cell types. J. Neurosci. 13, 981-989.
Veraart, J., Poot, D.H., Van Hecke, W., Blockx, I., Van der Linden, A., Verhoye, M., Sijbers, J., 2011. More accurate estimation of diffusion tensor parameters using diffusion kurtosis imaging. Magn. Reson. Med. 65 (1), 138-145.

Veraart, J., Novikov, D.S., Christiaens, D., Ades-Aron, B., Sijbers, J., Fieremans, E., 2016. Denoising of diffusion MRI using random matrix theory. Neuroimage 142, 394-406.

Wood, E.T., et al., 2012. Investigating axonal damage in multiple sclerosis by diffusion tensor spectroscopy. J. Neurosci. 32 (19), 6665-6669.

Zuchero, J.B., Barres, B.A., 2015. Glia in mammalian development and disease. Development 142 (22), 3805-3809. 